This item was submitted to Loughborough's Research Repository by the author.

Items in Figshare are protected by copyright, with all rights reserved, unless otherwise indicated.

\title{
Systems thinking-based risk assessment methods applied to sports performance: a comparison of STPA, EAST-BL, and Net-HARMS in the context of elite women's road cycling
}

\section{PLEASE CITE THE PUBLISHED VERSION}

https://doi.org/10.1016/j.apergo.2020.103297

\section{PUBLISHER}

Elsevier

\section{VERSION}

AM (Accepted Manuscript)

\section{PUBLISHER STATEMENT}

This paper was accepted for publication in the journal Applied Ergonomics and the definitive published version is available at https://doi.org/10.1016/j.apergo.2020.103297.

\section{LICENCE}

CC BY-NC-ND 4.0

\section{REPOSITORY RECORD}

Hulme, Adam, Scott McLean, Clare Dallat, Guy H Walker, Patrick Waterson, Neville A Stanton, and Paul M Salmon. 2020. "Systems Thinking-based Risk Assessment Methods Applied to Sports Performance: A Comparison of STPA, EAST-BL, and Net-harms in the Context of Elite Women's Road Cycling". Loughborough University. https://hdl.handle.net/2134/13650020.v1. 
Systems thinking-based risk assessment methods applied to sports performance: A comparison of STPA, EAST-BL, and Net-HARMS in the context of elite women's road cycling

Adam Hulme ${ }^{* 1}$, Scott Mclean ${ }^{1}$, Clare Dallat ${ }^{1}$, Guy H. Walker ${ }^{2}$, Patrick Waterson ${ }^{3}$, Neville A. Stanton $^{4}$, Paul M. Salmon ${ }^{1}$

\section{Contact details}

*Corresponding author: (ahulme@usc.edu.au)

${ }^{1}$ Centre for Human Factors and Sociotechnical Systems, Faculty of Arts, Business and Law, University of the Sunshine Coast, Sippy Downs, Queensland, 4558, Australia

${ }^{2}$ School of the Built Environment, Heriot-Watt University, Scotland, EH14 4AS, United Kingdom

${ }^{3}$ Human Factors and Complex Systems Group, Design School, Loughborough University,

Leicestershire, LE11 3TU, United Kingdom

${ }^{4}$ Transportation Research Group, Faculty of Engineering and Physical Sciences, University of Southampton, Southampton, SO16 7QF, United Kingdom 


\begin{abstract}
There is increasing interest in applying systems Human Factors and Ergonomics (HFE) methods in sport. Risk assessment (RA) methods can be used identify risks which may impact the performance of individual athletes, teams, and overall sports systems; however, they have not yet been tested in sport. This study sets out to apply and compare three systems thinking-based RA methods in the context of elite sports performance and report on the frequency and types of the risks identified. The Systems-Theoretic Process Analysis (STPA) method, the Event Analysis of Systemic Teamwork Broken Links (EAST-BL) method, and the Networked Hazard Analysis and Risk Management System (Net-HARMS) method were applied to elite women's road cycling to identify all the credible risks that could degrade optimal team performance. The findings demonstrate that all three methods appear to provide useful results in a context other than safety, and that multiple risks threatening the performance of the cycling team were identified. Whilst the frequency and types of risks differed across the methods applied, there are additional theoretical, methodological, and practical implications to be considered prior to the selection and use of systems thinking-based RA approaches. Recommendations and directions for future HFE and sports science research are discussed.
\end{abstract}

Keywords: Risk assessment, STPA, EAST-BL, Net-HARMS, sports performance, cycling 


\subsection{Introduction}

Risk assessment (RA) is an important area of Human Factors and Ergonomics (HFE) work whereby analysis methods are used to proactively identify risks that could hinder safety and performance. The outputs of such approaches are used to support organisations in developing appropriate and effective risk controls. However, whilst a large body of applied scientific research has attempted to understand the cause of adverse incidents from a 'systems thinking' perspective (Hulme et al. 2019a; Salmon et al. 2017a; Stanton et al. 2019a), there has been relatively little attention given to the development and use of systems thinking-based RA and safety management approaches (Dallat et al. 2017). To address this challenge, HFE researchers have proposed new systems thinking-based RA methods (Stanton et al. 2017a; Walker et al. 2017). Two recently developed RA methods are the Event Analysis of Systemic Teamwork Broken Links (EAST-BL) method (Stanton and Harvey 2017), and the Networked Hazard Analysis and Risk Management System (Net-HARMS) method (Dallat et al. 2018). These methods are multifaceted and scalable, affording risk assessors and analysts the scope to examine relationships and interactions among agents and factors from across entire sociotechnical systems. The other existing systems thinking-based RA approach that predates EAST-BL and NetHARMS is the Systems-Theoretic Process Analysis (STPA) method (Leveson 2011). Regarding incident analysis, STPA challenges older theories of reliability and safety (e.g., component failures, human error), instead supporting a holistic perspective that accounts for interactions among human and non-human factors across multiple system levels (Rising and Leveson 2018). As all three methods align with contemporary accident causation models (Dallat et al. 2018), it is our view that they represent contemporary, state-of-the-art RA approaches.

Alongside this evolution of RA methods, there is increasing interest in the application of HFE methods for the purposes of understanding and optimising both recreational and elite sports performance (Hulme et al. 2019b; Salmon and Macquet 2019; Salmon et al. 2020). In the area of systems HFE specifically, in-depth models of overall sports organisations and systems have been developed to identify factors that influence athlete and team performance (Hulme et al. 2019c; Mclean et al. 2019a). Whilst various systems HFE methods have been applied (Hulme et al. 2019b), RA 
methods have not yet been used in the sports context. In addition, the notion that safety theory and RA methods can be usefully applied to understand and optimise sports performance has not been tested, despite proponents arguing the case (Hulme et al., 2019b). Specifically, it is argued that such methods offer a potentially useful approach for understanding and responding to threats to performance at the athlete, team, and/or sports organisational levels. Accordingly, there is an outstanding need to: (i) formally test available systems thinking-based RA methods for sports applications; and, (ii) determine which state-of-the-art method is most suited to identifying risks that could degrade optimal sports performance.

Whilst STPA, EAST-BL, and Net-HARMS were each developed in consideration of a systems thinking philosophy (Dallat et al. 2017; Dallat et al. 2018; Leveson 2004; Stanton and Harvey 2017), they are fundamentally different in terms of their methodologies and analytical approach. STPA attempts to identify control and feedback failures based on a control structure model of the system, whereas EAST-BL uses task and social networks to identify potential failures in the communication of information between tasks and agents. Conversely, Net-HARMS focuses on the identification of emergent risks according to a description of the interacting tasks and activities undertaken within a system. With these differences in mind, knowledge around which RA method to select and use in practice, as well as for what problem(s) they are to be applied, is useful information for HFE researchers and practitioners who may have an interest in forecasting performance risks within the sports sciences. Given the relative novelty of EAST-BL and Net-HARMS along with the limited domains to which they have been applied to date (e.g., Dallat et al. 2018; Lane et al. 2019; Stanton and Harvey 2017), there is a need to formally test, evaluate, and report on their use in the HFE literature (Stanton 2016).

The purpose of this study is to apply STPA, EAST-BL, and Net-HARMS in the context of elite sports performance and report on the frequency and types of the risks identified. The aim of all three analyses is to identify as many credible risks as possible across the elite women's 'cycling race system' that could degrade optimal cycling team performance. A secondary objective is to compare and contrast each method in terms of their core methodological features, as well as comment on their 
accessibility and usability in HFE sports science practice. This includes reviewing the prerequisite materials, methods, and models that are needed to support the application of the RA evaluations. The application of new and contemporary systems thinking-based RA analyses may have implications for both sport and non-sporting domains, particularly work contexts and organisational systems that are high-risk and safety-critical in nature.

\subsection{The emergence of systems HFE in sport}

Before providing an overview of STPA, EAST-BL, and Net-HARMS, it is worth noting that there has been a newfound interest in the use of systems HFE methods and approaches in sport. Whilst physical and cognitive ergonomics have tended to be the focus of HFE in sport, covering topics such as the design of sports equipment, cognitive load, and naturalistic decision making (Atkinson and Reilly 1995; Hulme et al. 2019b; Macquet et al. 2015; Macquet and Fleurance 2007; Reilly and Lees 1984; Salmon and Macquet 2019; Salmon et al. 2020), systems HFE applications have seldom been applied until more recently (Hulme et al. 2019b). Systems HFE is aimed at understanding, modelling, and optimising the functioning of sports systems, including their organisational structures, policies, and processes. From a sports performance and injury management perspective, systems HFE attempts to consider the whole sports system, or multiple interacting elements of it, as the primary unit of analysis (Hulme et al. 2019b).

Recent systems HFE studies in sport have modelled the elite football (soccer) match system using Work Domain Analysis (WDA) (Naikar 2005; Naikar et al. 2006) to better understand the interactions among multiple components underpinning optimal player and team performance (McLean et al. 2017a). Other studies within the football context have applied network analysis - a popular approach for analysing communications in HFE - to understand the features and structures of successful passing networks preceding goals scored during major international championships (McLean et al. 2019b; McLean et al. 2017b). In other sporting areas, researchers have developed a control structure model (Leveson 2004) of the Australian recreational distance running system to identify systemic leverage points to guide the implementation of sustainable injury prevention interventions (i.e., those that reside beyond the individual athlete and behavioural levels) (Hulme et al. 2017a; Hulme et al. 
2017b). Likewise, popular HFE models such as the Risk Management Framework (RMF)

(Rasmussen 1997) have been used to explore the perceived responsibilities for identifying and treating sports-related concussion in rugby union (Clacy et al. 2019). Finally, different phases from Cognitive Work Analysis (CWA) (i.e., WDA, Social Organisation and Cooperation Analysis; SOCA) (Jenkins et al. 2017; Vicente 1999) have been applied to model elite netball organisations (Hulme et al. 2019c) and identify key components of match performance to complement traditional sports science research (Mclean et al. 2019a).

Against this backdrop of emerging systems HFE methods in sport, there is an opportunity to extend the sports research agenda by exploring what STPA, EAST-BL, and Net-HARMS (Dallat et al. 2018; Leveson 2011; Stanton and Harvey 2017) can add to the field. This opportunity serves as justification for the current study and may form the initial foundation of a complementary systems thinking-based RA and evaluation theme within the sports sciences.

1.2 Overview of systems thinking-based RA methods

\subsubsection{The Systems-Theoretic Process Analysis (STPA) method}

An overview of STPA requires a brief introduction to the Systems-Theoretic Accident Model and Processes (STAMP) framework (Leveson 2004). This is because STAMP forms the basis of the STPA approach and is a necessary component to support the RA analysis. STAMP builds directly on the control-theoretic foundation proposed by Rasmussen and Svedung's (2000) sociotechnical model of system operation and so many similarities can be found. First, STAMP applies the controltheoretic approach across all levels of a system hierarchy, down to and including the technical and physical work context. Second, the model emphasises the role of upstream processes in preventing the occurrence of hazardous system states, including events and activities at the legislative, regulatory, and company management levels. Third, in addition to system operation, STAMP models the design and safety requirements around system development to support proactive RA and safety management. Forth, the inclusion of a broad range of control and feedback types in STAMP demonstrates that system performance and safety is influenced by policies, procedures, shared values, 
and other aspects of the surrounding social and organisational culture (i.e., the onus is not placed solely on individuals or indeed hard physical and engineered controls). Fifth and finally, STAMP includes a classification system of control flaws (i.e., Causal Analysis based on STAMP (CAST) for accident analysis purposes), as well as a system of hazard identification (i.e., STPA) to support RA activities (Leveson 2011). With the above aspects in mind, STAMP promotes the view that adverse incidents occur when external disturbances, component failures, and/or dysfunctional interactions among both human and non-human system entities are not adequately managed by the system's control structure (note: see Leveson (2004) for a generic control structure illustration and further explanation). From a sports performance perspective, a STAMP model of the elite cycling race system, including a range of control and feedback mechanisms, is later presented in Figure 1 (section $3.1)$.

The primary reason for developing the STPA (Leveson 2011) method was to cater to the systemic focus offered by STAMP, including a range of new control types and factors that were not handled by older techniques (e.g., Fault Tree Analyses (FTA), Hazard and Operability Study (HAZOP)). For instance, given its connection to STAMP, STPA can account for design errors, software flaws, component interactions, cognitively complex human decision-making errors, and social, organisational, and management factors that could conceivably lead to a loss event (Leveson 2011). STPA can be used at any stage of a systems lifecycle and involves identifying the potential for inadequate control of the system that could lead to a hazardous state. Hazardous states result from the inadequate control or the enforcement of safety constraints, which can occur because:

i. A control or feedback action required for safety is not provided or followed;

ii. An unsafe control or feedback action is provided;

iii. A potentially safe control or feedback action is provided too early or too late, that is, the time or in the wrong sequence; and,

iv. A control or feedback action required for safety is stopped too soon or applied too long. 
Although not included above, the described risks following the identification of absent or unsafe control actions is used to inform a second STPA step; namely, identifying the causes of the unsafe control actions. The use of STPA helps analysts to gather information about how existing system constraints could fail to ensure their appropriate and ongoing enforcement.

\subsubsection{The Event Analysis of Systemic Teamwork Broken Links (EAST-BL) method}

EAST-BL is an extension of the EAST framework which draws on the theory of distributed cognition to support the evaluation of performance in complex systems (Stanton et al. 2018; Stanton et al. 2008; Stanton et al. 2019b). The EAST methodology was first applied in the early 2000s to analyse command and control activity in a diverse range of domains (e.g., emergency services, military, defence) (Salmon et al. 2005; Stanton et al. 2008). Over the last decade, EAST has been further developed, refined, and streamlined. More recent studies have presented a shortened version of EAST that uses Hierarchical Task Analysis (HTA) and/or the raw data obtained from field-based observations and existing literature to directly develop three networks: (i) a task network; (ii) a social network; and, (iii) an information network (Banks and Stanton 2019; Lane et al. 2019; Stanton et al. 2018; Stanton 2014). Task networks provide a summary of the interdependence of activities performed within a system (i.e., the relationships between tasks). Social networks analyse the structure of the system and the communications taking place between agents (i.e., who/what interacts and communicates with whom/what). Information networks describe how information and knowledge is distributed across different tasks and system agents (i.e., what information is transmitted at points in time). In terms of sports performance, a task, social, and information network of the cycling race system can be viewed in Figure 2, 3, and 4 (section 3.2). The three EAST networks can then be combined to form a composite network demonstrating how different agents utilise information to conduct and undertake a range of tasks (Salmon et al. 2014; Salmon et al. 2018; Stanton et al. 2018; Stanton 2014). EAST can be applied to all system levels, including individual human-machine interactions, organisations operating highly autonomous systems, and a multi-layered network system (Stanton 2014). Stanton \& Harvey (2017) recently extended the EAST method to consider the potential risks that emerge when information is not transacted between agents and tasks. 
Underpinning EAST-BL is the notion that accidents and adverse incidents are caused due to failures safety is therefore a matter of understanding how information is communicated between various tasks and agents, including the likely adverse effects that result from 'breaking' links within the EAST networks. To conduct an EAST-BL analysis, the relationships between the linked tasks and agents in the task and social networks are examined to determine what would happen if the requisite information were not to be transacted. Notably, the EAST information network is not subject to an EAST-BL analysis as information does not fail in isolation, rather it is the failure to use or communicate information correctly between tasks and agents. In sum, EAST-BL offers a systemsbased, structured, and systematic RA approach to the identification of potential communication and information failures within complex systems (Stanton and Harvey 2017).

\subsubsection{The Networked Hazard Analysis and Risk Management System (Net-HARMS) method}

Net-HARMS (Dallat et al. 2018) was developed based on the findings from a review of existing RA approaches which indicated that a majority of current methods are not aligned with contemporary models of accident causation and do not support the identification of risks across work systems (Dallat et al. 2017). Net-HARMS provides two key advances over existing RA methods. First, it enables analysts to identify risks across the overall system, as opposed to 'sharp end' risks only. Second, Net-HARMS enables analysts to identify emergent risks that arise when different risks combine and interact with one another.

Net-HARMS is structured around, and incorporates, both HTA (Annett et al. 1971; Kirwan and Ainsworth 1992; Shepherd 1998; Stanton 2006) and the Systematic Human Error Reduction and Prediction Approach (SHERPA) (Embrey 1986). A recognised limitation of SHERPA includes a focus on tasks and errors at the 'sharp-end' of work systems as opposed to the tasks and risks that can occur at higher system levels (e.g., legislative, regulatory, organisational) (Dallat et al. 2018; Stanton et al. 2017a). Consequently, in developing Net-HARMS, both HTA and SHERPA were extended and modified to describe the goals, tasks, operations, and plans required across entire work systems, as well as to support the identification of system-wide risks and emergent risks (Dallat et al. 2018). In 
addition, 'error modes' in the SHERPA taxonomy was aptly renamed to 'risk modes' for NetHARMS whilst being further refined to reflect the needs of a systems analysis. The Net-HARMS risk modes taxonomy contains three categories and 10 risk modes:

1. Task

i. T1: Task mistimed

ii. T2: Task omitted

iii. T3: Task completed inadequately

iv. T4: Inadequate task object

v. T5: Inappropriate task

2. Communication

i. $\quad$ C1: Information not communicated

ii. $\quad$ C2: Wrong information communicated

iii. C3: Inadequate information communicated

iv. C4: Communication mistimed

3. Environmental

i. E1: Adverse environmental conditions

Methodologically, NET-HARMS is similar to a traditional SHERPA analysis, however differences include a broader analytical scope in terms of the HTA output, as well as the use of a task network to facilitate the identification of emergent risks. Applying Net-HARMS involves first developing an HTA describing the overall system under analysis - in this case the cycling race system. The HTA is then converted into a task network (Figure 2, section 3.2) which shows the core tasks and the relationships between tasks. Task risks are identified by applying the Net-HARMS risk mode taxonomy (as above) to each task node within the task network. Following task risk identification, the risk mode taxonomy is applied once more to identify emergent risks that arise when task risks interact with one another.

1.3 Recap of study aims 
In consideration of the emergence of HFE applications in sport and the recent development of novel HARMS in the context of elite sports performance and report on the frequency and types of the risks identified. RA methods are traditionally applied in safety-critical domains, and so evaluating their use in a sports performance context will demonstrate their capability in a new and upcoming HFE area. A secondary objective is to report on the core methodological features of all three methods, as well as comment on their accessibility and usability in HFE sports science practice.

\subsection{Methods}

This RA analysis focuses on, and is applicable to, cycling road races more generally. However, the models and networks described were developed based on two elite women's road races undertaken as part of the 2016 Australian Subaru National Road Series (NRS). The networks were developed as part of a study in which EAST was used to assess teamwork in a women's elite cycling team (Salmon et al. 2017b). An overview of cycling road racing and the method is provided.

\subsection{Overview of elite cycle racing}

Elite NRS cycle races range in length from 50 kilometres $(\mathrm{km})$ to $130 \mathrm{~km}$ and involve teams of four to five riders. Each team has a protected rider (PR) who is selected based on overall NRS points standing. The remaining team riders act in a domestique or support capacity to ensure that the PR finishes each race in the best position and time possible. The domestiques' roles include shielding the PR from headwinds and performing a 'lead out' to optimise sprint finishing, among others. During a race, the riders from each team self-organise to form a peloton or larger group of cyclists as a way to conserve energy through zones of reduced air resistance. Throughout the race, riders may 'attack' to break away from the peloton. In response, other teams may 'bridge across' or wait until a later point in time to 'bring back the break' (e.g., upon reaching a hilly section of the course). Alternatively, the peloton may stay together resulting in a bunch sprint finish. Towards the end of the race, domestiques who have fulfilled their roles of protecting the PR retire due to fatigue. Following closely behind the peloton is a convoy of vehicles comprising the commissaire, race officials, medical support, the 
media, and team personnel. The commissaire controls the race and convoy, communicating with the riders via loudspeaker and with other vehicles via UHF radio. Each team's support vehicle contains a Director Sportif (DS) or team leader, mechanic, spare parts (e.g., tyres, wheels) and nutritional supplies. Support vehicles provide mechanical services and ergogenic aid to team riders and offer tactical guidance and verbal encouragement. When viewed together, the peloton and convoy form a complex, highly dynamic system including both human agents (e.g., domestiques, PR, DS, commissaire) and non-human factors (e.g., environment, bikes, vehicles, communication devices, computers) that exhibit non-linear interactions, multiple control and feedback loops, loose and tight coupling, rapid decision making, and emergent properties and outcomes (Salmon et al. 2017b). Given the complexity and unpredictability of elite cycle racing, RA approaches that assess and predict the risks affecting optimal sports performances are warranted.

\subsection{Definition of cycling performance}

Cycling team performance was defined in the present study as the ability of cycling team members to work cooperatively to achieve the shared goal of protecting the PR and winning the race. Risk credibility was determined based on the potential of a given risk to detrimentally affect the ability of the cycling team to achieve their shared goal of winning the race.

\subsection{Participants}

Participants were members of an elite women's Australian NRS cycling team. The sample included five riders, one DS, and one mechanic. All participants were experienced in elite cycling and had been actively involved throughout the 2016 season. Institutional ethics approval was granted by the University of the Sunshine Coast Human Ethics Research Committee (Project ID: A/16/828).

\subsection{Materials}

Two members of the research team (CD, PS) observed the race from within the cycling team's support vehicle. Go Pro cameras were used to video record the races and a Dictaphone was used to record the verbal communications occurring within the team support car. Dictaphones were also used to record team race planning meetings, the post-race Critical Decision Method (CDM) interviews 
(Klein et al. 1989), and post-race team debriefs. The interview transcripts were transcribed in word information networks were created using diagramming software (Microsoft Visio) (the methods used around network development can be viewed in section 2.6.2).

\subsection{Procedure}

The cycling team were provided with an overview of the study, its aims, and the data collection methodology prior to the first race of each event. The research team travelled to each race with the cycling team and observed the warm-up and pre-race discussions. The Go Pro cameras and Dictaphones were placed within the support vehicle. Fieldnotes were taken during the race regarding the team's performance and tactics, as well as the interactions occurring between the DS, the commissaire, and riders. During periods of low workload, individual rider and team behaviours were discussed with the DS. Following the race, the research team audio recorded the team's post-race debrief and planning session for the next race. At the conclusion of the planning and debrief sessions, CDM interviews with each rider regarding the concluded race were conducted. The CDM utilised cognitive probes adapted for the cycling context to elicit information about how the riders formulated their decision-making strategies. Following the interviews, each rider completed a social network diagram to show who and what they interacted with during the race. This involved presenting them with a diagram showing all agents (i.e., riders, other riders, DS, commissaire, cycling computer) and asking them to rate their level of communication with each during the race on a scale of low $(<10$ communications), medium (between 10 and 20 communications) and high (>20 communications).

\subsection{RA methods procedures}

This section outlines the procedures undertaken for the STPA, EAST-BL, and Net-HARMS analyses. Three separate analysts (AH, SM, PS) independently conducted each RA analysis to ensure that the identification of risks was not influenced by the results of one of the other approaches. A fully detailed step-by-step methodological guide for each RA approach, covering the development of prerequisite materials through to the conduct of analyses, can be found elsewhere for STAMP 
(Leveson, 2004; Leveson et al. 2009) and STPA (Leveson, 2011; Leveson and Thomas, 2018), EAST

(Stanton et al. 2018; Stanton et al. 2008; Stanton et al. 2019b) and EAST-BL (Lane et al. 2019; Stanton and Harvey 2017), and Net-HARMS (Dallat et al. 2018).

\subsubsection{Procedure for STPA}

A STAMP control structure model of the cycling race system was developed by three of the authors, two of which have previous experience in developing STAMP control structures (Hulme et al. 2017b; Salmon et al. 2016). To ensure a fair comparison between the other two RA methods, the control structure model contained the same human agents as found in the task network which was subsequently used as the basis for the EAST-BL and Net-HARMS analyses (Figure 2). Likewise, the control and feedback action descriptions between agents were identified after reviewing the EAST-BL and Net-HARMS task networks (see section 2.6.2). One author (PS) used spreadsheet processing software (Microsoft Excel) and applied the four-item STPA taxonomy (section 1.2.1) to each potentially hazardous control and feedback action as described in the control structure model.

\subsubsection{Procedure for EAST-BL}

The three EAST networks were constructed as follows. The task network was developed based on the observations of both races, facilitated by a review of the video data and the interview transcripts. Tasks were deemed to be related to one another if the conduct of one task influenced, was undertaken in combination with, or was dependent upon, another task (Stanton et al. 2018). A draft version of the task network was reviewed and refined by the cycling team during the NRS event. Construction of the social network (Figure 3) involved transforming the social network diagrams obtained following the races into a network detailing the number of interactions between the cyclists and other agents. The information network (Figure 4) was constructed through content analysis of the riders' interview transcripts. Specifically, the riders' responses to the CDM questions: 'What were your specific goals for the race?', 'What information were you using throughout the race? '; and, 'What was the most important piece of information', were analysed by a member of the research team (PS). For example, from the sentence, 'I was checking my position in the peloton', the related concepts 'Checking', 
'Position', and 'Peloton' were extracted. Once the information network was constructed, concepts social network used what type of information element. The development of the three EAST networks was the first step in being able to conduct the EAST-BL analysis.

For the purpose of conducting the EAST-BL analysis, one analyst (SM) used spreadsheet processing software (Microsoft Excel) to perform three different phases: (i) a task-information description (i.e., what information was being used during each task); (ii) a social-information description (i.e., which agents used what type of information); and, (iii) the EAST-BL analysis itself which involved taking phase (i) and (ii) above and noting the potential risks should an information element from a linked task or agent fail to be transmitted.

\subsubsection{Procedure for Net-HARMS}

The EAST task network was used to carry out the Net-HARMS analysis (Figure 2). The first phase of the Net-HARMS analysis was to identify the risks associated with each task in the task network. To do this, one author (AH) used spreadsheet processing software (Microsoft Excel) and applied the NetHARMS risk modes taxonomy (section 1.2.3) to each task, drawing on domain expertise to determine credible risks based on the definition of cycling performance (section 2.2). Where relevant, the tasks involving multiple agents (e.g., DS, commissaire, riders) were analysed separately. This decision represents a refinement of the original Net-HARMS method (Dallat et al. 2018) yielding considerably more task and emergent risks by stratifying tasks by agent type. The reason for this stratification decision and the modification to the method was due to fact that some tasks were performed by multiple agents. For example, the task 'Monitor other riders' is performed by the commissaire, DS, and riders; however, the nature of the behaviour associated with these agent types is different. The commissaire may monitor other riders for safety, the DS for strategic team monitoring purposes, and the riders for immediate opposing team actions that might directly impact performance. A description of a specific risk was provided along with the Net-HARMS risk mode, and multiple risk modes for a given task were identified where relevant. 
The second phase of the analysis involved the identification of emergent risks. Emergent risks risks identified during phase one. The emergent risk identification process involved reviewing the task network and making a note of which tasks had a dependency on another task. The directionality of the relationship between tasks was recorded along with a description of the initial risk modes and descriptions for the tasks identified in the first phase. The Net-HARMS risk modes taxonomy was reapplied, however this time the emergent risk mode and emergent risk description were based on asking the question: how could task A (e.g., 'Race planning and tactics') degrade related task B (e.g., 'Warm up') if the former task was mistimed/omitted/completed inadequately (and so on). Again, multiple risk modes for a given related task were identified where relevant. The emergent risk identification component of Net-HARMS produces considerably more risks relative to phase one due to the multiplicative effect of combining risks modes.

\subsection{Results}

\subsection{STAMP control structure and STPA}

The control structure model of the cycling race system is presented in Figure 1. It includes control and feedback actions linking agents (e.g., riders, DS, team mechanic, commissaire) and factors (e.g., bike, convoy, vehicles) across the system.

<insert Figure 1 about here>

A total of 211 risks were identified following the application of STPA. Broken down by the four STPA hazardous types of behaviour: 48 risks were identified for 'action or feedback required but not provided'; 47 risks were identified for 'unsafe action or feedback provided'; 59 risks were identified for 'incorrect timing or order'; and, 57 risks were identified for 'stopped too soon/applied too long'. Table 1 is an excerpt of the STPA analysis showing a selection of risks identified across the four 
categories. A description of the control and feedback actions between agents from the control structure model, and the risks that were deemed to be associated are presented. The complete STPA analysis is appended as electronic supplementary material A.

<insert Table 1 about here>

\subsection{EAST networks and EAST-BL}

The task network of the cycling race system is presented in Figure 2. The circular nodes within Figure 2 represent the distinct tasks undertaken immediately prior to and during the road races. The coloured rings around each node represent the agents in the cycling system who undertake and perform each task. Both individual and shared tasks are identified. For example, the task 'Communicate with other riders' is undertaken by team riders within the peloton (e.g., asking for assistance to initiate a break), by the DS in the support vehicle (e.g., communicating tactics with team riders), and by the commissaire (e.g., informing other riders in the peloton and the support vehicles of the time gap between a breakaway rider and the peloton).

\section{<insert Figure 2 about here>}

The EAST social network is presented in Figure 3. The circular nodes represent agents (e.g., riders, support vehicle, bicycle computers) and the arrows between them represent the extent to which the agents communicated with one another during the race. The social network shows the structure of communications throughout the road race. In this case the PR and domestique rider two are the most connected in terms of incoming and outgoing communications. In addition, both the PR and domestique rider two have a greater frequency of communications with other agents. 
<inset Figure 3 about here>

The information network is presented in Figure 4. Within the network, the nodes represent individual information elements whereas the links between the nodes represent relationships between information (e.g., 'Computer' displays 'Speed').

<insert Figure 4 about here>

A total of 404 risks were identified across both EAST-BL phases (i.e., 345 for the task-information and 59 for social-information phases). Table 2 is an excerpt of the EAST-BL analysis for multiple tasks from the task-information phase of the analysis. The complete EAST-BL analysis is appended as electronic supplementary material B.

<insert Table 2 about here>

\subsection{Net-HARMS}

A total of 157 task risks were identified following phase one of Net-HARMS. For the emergent risk phase, a total of 1,936 risks were identified (i.e., there were 12.8 times more emergent risks, or a $1133 \%$ increase) (Figure 5). Both phases therefore produced 2,093 risks. Figure 5 indicates that there were a greater number of emergent task risks relative to emergent communication risks, with T1 (i.e., task mistimed; 474 risks), and T3 (i.e., task completed inadequately; 386 risks) yielding the highest frequencies. No emergent E1 risks (i.e., adverse environmental conditions) were identified, owing to 
the nature of this mode being more of an influence on other tasks than a risk that is generated through interaction. The process of identifying emergent risks occurred for all tasks within the task network.

<insert Figure 5 about here>

Table 3 provides an excerpt of the Net-HARMS emergent risk analysis. The initial task, agent(s), risk mode, and risk descriptions, as viewed in the first four columns, were carried over from Net-HARMS phase one and applied in phase two. The eight emergent risks presented in Table 3 represent $.004 \%$ of the total emergent risks identified. The complete Net-HARMS analysis is appended as electronic supplementary material C.

<insert Table 3 about here>

\subsection{Risk frequency comparisons between EAST-BL and Net-HARMS}

Due to the fact EAST-BL and Net-HARMS both use the same task network, it was possible to perform a side by side comparison of the identified risk frequencies. Figure 6 transforms the task network into an adjacency matrix plot visualising the frequency with which risks were identified for the task-information phase of the EAST-BL analysis, and the emergent risk identification phase for Net-HARMS. Regarding EAST-BL, the task 'Race planning and tactics' produced the highest aggregated risk frequency, with 92 risks identified among 10 related tasks. Twenty-two risks were identified from the task 'Update convoy' to the task of 'Update riders'. The mean number of risks that were deemed to be associated with each related set of tasks following the EAST-BL analysis was $5.3(S D=3.5)$. In terms of Net-HARMS, the task 'Monitor speed/cadence/distance etc' produced the highest aggregated emergent risk frequency, with 443 risks identified among seven related tasks. Seventy risks were identified from the task 'Communicate with team mates' to the task 
'Establish/maintain appropriate position in peloton'. The mean number of risks that were deemed to be associated with each related set of tasks following the Net-HARMS analysis was $31.7(S D=20.2)$.

<insert Figure 6 about here>

Figure 7 visualises the differences between aggregated risk frequencies for EAST-BL and NetHARMS among tasks with outgoing relationships. The mean number of risks and emergent risks identified in the EAST-BL and Net-HARMS analyses for these tasks was $20.3(S D=23.0)$ and 113.9 $(S D=153.8)$, respectively.

<insert Figure 7 about here>

\subsection{Discussion}

The purpose of this study was to apply STPA, EAST-BL, and Net-HARMS in the context of elite sports performance and report on the frequency and types of the risks identified. The aim of all three analyses was to identify as many credible risks as possible across the elite women's cycling race system that could degrade optimal cycling team performance. A secondary objective was to compare each method in terms of their core methodological features, as well as comment on their accessibility and usability in HFE sports science practice. There were two main reasons for undertaking the RA comparative analysis. First, there is a growing interest around the application and utility of systems HFE methods and approaches in sport (Hulme et al. 2019b; Salmon and Macquet 2019; Salmon et al. 2020); however, the extent to which systems thinking based RA methods can provide useful outputs when used to identify performance-related risks has not yet been examined. From a theoretical perspective, the notion that safety-related HFE theory, developed specifically to help enhance safety, can also be used to optimise elite sports performance also requires exploration. Second, compared to 
STPA which is well established in the literature (e.g., Allison et al. 2017; Leveson 2011; Mahajan et al. 2017; Revell et al. 2019; Rising and Leveson 2018; Rokseth et al. 2018; Schmid and Stanton 2018; Schmid and Stanton 2019), EAST-BL and Net-HARMS required further formal and independent testing beyond the limited applications to date (Dallat et al. 2018; Lane et al. 2019; Stanton and Harvey 2017). This testing is important both for future HFE applications in the safety critical domains but also to support further HFE applications in sport. The following discussion is structured around the main findings and will outline several directions for future research.

\subsection{Frequency and types of risks identified}

STPA, EAST-BL, and Net-HARMS produced 211, 404, and 2,093 risks, respectively. Net-HARMS yielded the highest risk frequency due to the incorporation of an emergent risk phase. Indeed, 12.8 times more risks were identified in phase two relative to phase one. This focus on the identification of emergent risks is a distinct strength of Net-HARMS over any other systems-thinking based RA method available in the HFE and safety science literature (Dallat et al. 2017; Dallat et al. 2018). EAST-BL also includes two phases; a task-information and social-information phase. Depending on the size of the task, social, and information networks in terms of the number of nodes and connections, the granular analysis offered by EAST-BL may explain why almost twice as many risks were identified compared to STPA. For example, multiple pieces of information were deemed to be relevant and thereby 'broken' for many of the linked tasks and agents. This includes 22 identified risks from the task 'Update convoy' to 'Update riders' covering information elements such as 'Energy', 'Breaks', ‘Tactics', ‘Attacks', 'Protected rider', 'Course', and 'Weather'; and, 15 identified risks from the task 'Race planning and tactics' to 'Sprint finish' covering information elements such as 'Speed', 'Power', 'Time gap', 'Goals', and 'Position'. Of further interest is how each of the risks were identified and categorised. For example, STPA includes four hazardous types of behaviour; EAST-BL was designed to be free of a taxonomy; and, Net-HARMS uses a 10-item risk modes taxonomy that is applied multiple times to support the identification of emergent risks. These taxonomic differences (or lack thereof) may also explain the frequency of the risks identified using each method. Specifically, whilst STPA offers four potential risks per control or feedback action, 
Net-HARMS offers 10. Most revealing is the breakdown of risk frequencies by related tasks whereby in many cases a considerable difference between Net-HARMS and EAST-BL can be observed (Figure 6). In light of these differences, it is these authors' opinion that so long as the identified risks are deemed to be credible; that is, they could reasonably occur in practice, preference should be given to a more comprehensive RA approach that identifies as many risks as possible. In this regard, and notwithstanding the time taken to complete analyses - a caveat to be discussed in section 4.3 - NetHARMS was the method most capable of accounting for the highest number of potential risks within the cycling race system.

Regarding the types of the risks identified, all three methods appear to generate useful insights about how performance could be degraded in the elite cycling race system. This suggests that RA methods developed specifically to identify risks in safety-critical domains and work contexts can produce relevant outputs when used to identify risks to athlete, team, and system performance. For example, Tables 1, 2, and 3 are excerpts of the complete analyses that encompass a range of different conditions and scenarios that could hinder and impair optimal cycling team performance. Each analysis contains risk descriptions that are relevant to, and can be used by, all agents within the cycling race system, including the riders, the DS, and support crew. Importantly, the findings also provide further evidence that contemporary safety and accident causation theory apply equally well to sports performance (Hulme et al. 2019b). For example, according to Rasmussen's (1997) RMF, networks of factors across work systems interact to create accidents. In the present study risks were identified across the sports system relating to equipment and technology (e.g., bikes, power meters, GPS devices), nutrition and ergogenic aids, race planning, tactics and strategy, specific features of the terrain and topography, rider behaviours (e.g., attacking, surging), opposing team behaviours, and race management and governance.

A closer look at the types of risks described across all three methods points to a number of further subtle but important differences. For example, three of the 14 EAST-BL risks identified from the task 'Race planning and tactics' to the task 'Form break' included: (i) not being aware of the course profile, including its topographical features; (ii) not accounting for the plan around when a break is to 
occur (i.e., timing among the team not necessarily opponents); and, (iii) having no feedback around power output which is used to regulate prolonged bouts of physical effort. Taken together, these risks suggest that if members of the cycling team attempt to form a break prior to, for instance, a downhill section of the course and also lack an objective understanding of their power output, then there is a possibility that a breakaway is not going to be effective. Indeed, opponent teams could use the course profile and downhill sections to their advantage and 'bridge the gap' to the breakaway with relative ease. From the perspective of cycling team performance, these potential risks are useful for highlighting the importance of continually monitoring key indicators of race status, such as the position of other teams, course location, power, speed, time gaps, and distances. With regard to the same pair of tasks using Net-HARMS (i.e., 'Race planning and tactics' to 'Form break'), a total of 40 risks were identified; however, many of the risk descriptions were not as detailed as the 14 described with EAST-BL and were more general in nature. For example, whilst the Net-HARMS analysis still included specific risks that could degrade efforts to form a breakaway, such as not accounting for timing or the appropriate course location, there was equally no mention of power, speed, or opponent team positioning. This is a level of detail that EAST-BL can (and did) offer due to the provision of an information network that supports the RA analysis. Thus, whilst many more risks were identified using Net-HARMS, and even though many of those appear useful in and of themselves, the method may benefit from a more granular task network as well as an expert analyst who can add a suitable level of detail to the risk descriptions. Regarding STPA, the STAMP control structure described a range of different control and feedback actions among key agents within the cycling race system. As a result, the corresponding risk descriptions across the four hazardous types of behaviour were also specific to the control and feedback action(s) being examined. In a similar way to the networks associated with EAST-BL and Net-HARMS, the value and utility of a given STPA output is largely dependent on the quality and face validity of the STAMP control structure model, including the fidelity of the control and feedback actions described.

Overall, and given the high number of risks identified, a useful future direction involves collaborating with the cycling team to develop appropriate remedial measures and intervention strategies to address 
potential risks and optimise subsequent race performances. Alternatively, and depending on the expectations and goals of the project and overall clarity of the risk descriptions, HFE researchers and practitioners may have the option of handing over their RA analyses to decision-makers and key stakeholders who can prioritise and/or incorporate findings into future planning activities. In the case of the cycling RA analysis, key lessons and insights could be extracted by the riders and the DS and applied to the next race in the NRS series to enhance team performance moving forwards.

\subsection{Core methodological features of STPA, EAST-BL, and Net-HARMS}

An objective of this study was to compare all three methods according to their core methodological features. Following the RA evaluations, the three analysts who applied STPA, EAST-BL, and NetHARMS identified a set of theoretical and methodological constructs that were either shared or were considered unique to each method (Table 4).

<insert Table 4 about here>

Reflecting on the constructs in Table 4, a hierarchical control structure is not required to undertake an EAST-BL or Net-HARMS analysis. Conversely, a taxonomy is used in STPA and Net-HARMS but is not needed in EAST-BL. Social agents feature in all three methods, but only because the NetHARMS analysis stratified relevant tasks by agent type, representing a modification to the original approach (Dallat et al. 2018). Emergence is a concept that only Net-HARMS incorporates (i.e., specifically emergent risks), whereas all three methods have the capacity to include multiple system levels, as well as model relationships and interactions among factors (or tasks and agents). EAST-BL and Net-HARMS structure their analyses around tasks and activities, whereas STPA focuses on control and feedback actions linking human and non-human components across a system. The feedback construct in Table 4 refers not to a closed system of feedback in the sense that it has potential to amplify, achieve equilibrium, or dampen over time; rather, it is whether the RA methods include relationships and interactions that are two way or bidirectional. For example, in the context of 
elite cycle racing, it is possible to have a miscommunication of instruction from the DS to the riders and also from the riders back to the DS. A final consideration is how each of the RA methods use collected data to support the analysis. For example, STPA reflects a data-fit-model approach whereas EAST-BL and Net-HARMS are more aligned with a model-fit-data approach. This means EAST-BL and Net-HARMS are not constrained by pre-existing models or frameworks (i.e., Leveson 2004) and so the task, social, and information networks can be as large and/or as detailed as they need to be (Stanton and Harvey 2017). With that said, it is theoretically possible to describe hundreds or thousands of control and feedback actions across a highly detailed control structure model which would result in many different types of risks being identified when using the STPA method.

\subsection{Accessibility and usability of systems thinking-based RA methods}

HFE researchers and practitioners who work within risk management and safety-related roles are expected to engage in both proactive and reactive incident analysis activities. This includes forecasting and predicting adverse events before they occur, as well as analysing the cause of incidents after they have happened. As a result, knowledge and information to support the selection and use of HFE methods and models for RA and incident analysis will assist practitioners to undertake activities within their own professional roles, especially in consideration of the (often limited) time and resources available to them. After applying all three methods in this study to a comparable system, it is concluded that STPA can be applied relatively quickly, followed by EASTBL and then Net-HARMS. Although the time it took to undertake the analyses was not objectively quantified in discrete units of time, minutes or hours, it is fair to say that STPA was completed within a day, EAST-BL over two to three days, and Net-HARMS over a 60-day period. Notably, these approximated timeframes do not account for the materials, methods, or models that were first required to support the RA analyses.

Whilst the model-fit-data approach associated with EAST-BL and Net-HARMS may be considered advantageous, the development of task, social, and information networks equally necessitates many resources and a high level of HFE knowledge and expertise. Educational resources outlining the procedures around HTA and the construction of networks can be found elsewhere (Stanton et al. 
2018; Stanton et al. 2017a). In short, there are several required steps prior to the application of and system to be modelled; identifying suitable data collection procedures and undertaking data transcription; constructing task, social, and information networks (and composite networks where relevant); and, analysing networks with network analysis to produce density, sociometric status, and centrality metrics (Stanton et al. 2019b). Whilst EAST-BL requires many if not all of the steps above (i.e., composite networks and network analysis are useful albeit not essential), Net-HARMS can be undertaken following the development of a task network. There can be a considerable amount of preparation and work required to collect data and construct networks and models to support the EAST-BL and Net-HARMS analyses.

In terms of STPA, Leveson (2011) outlines a detailed step-by-step guide to support the development of a STAMP control structure, as well as instructions for undertaking the RA component. Developing control structure models, identifying human and non-human factors to populate the framework, and modelling control and feedback actions across the system involves a series of steps and prompts that are to be systematically followed (Leveson and Thomas, 2018). Whilst Net-HARMS produced the highest number of risks and given the potential scalability of EAST-BL to also yield a high frequency, it may be the case that the provision of a STAMP control structure the and relatively short timeframe associated with the application of STPA makes this method highly attractive in HFE practice. However, the main caveat being that, all things being equal (i.e., system descriptions, tasks, control and feedback actions are comparable across methods and analyses), STPA will identify fewer risks and cannot account for emergent risks (or risks that emerge when control failures interact with one another).

\subsection{Future directions and recommendations}

Several recommendations are proposed as a result of the work undertaken. The following points of discussion are not only applicable to sport and sports performance but are also directly relevant to the broader HFE discipline. First and foremost, the need for this work was attributable to the growing interest in the use of HFE methods and approaches that have the capacity to model the interactions 
among various factors within complex sports systems (Hulme et al. 2019b; Salmon et al. 2020). For decades sports science research, whether to enhance athletic performance or reduce the risk of injury, has been dominated by traditional epidemiological, clinical, and biomechanical studies (Hume et al. 2019b). Such studies are, however, generally used to identify and isolate a series of statistically significant biological and/or behavioural factors at the level of the individual athlete. As such, the resulting performance enhancing interventions and remedial measures are generally reductive and attempt to modify the physical properties and profiles of players, teams, and/or the immediate environment. The findings of this study, therefore, point not only to the need for further systems HFE applications in sport, but also a requirement to specifically extend the RA agenda and use novel HFE methods such as EAST-BL and Net-HARMS in other sporting areas. Doing so will further highlight that to fully optimise sports performance, it is necessary to model, describe, and understand the interactions among various agents (e.g., athletes, coaches, team support personnel), tasks and factors from across a well-defined sports system. Many of these risks and factors reside beyond the physical level of the individual athlete and are necessary to consider from a coaching standpoint when aiming to enhance sports performance from a more holistic perspective. From this it is concluded that there is a need to continue to apply systems-based RA approaches and HFE methods in other individual and team sports contexts to complement more traditional sports science research.

Second, the findings also suggest that it may be useful to apply RA methods in HFE applications seeking not to identify safety risks but instead to optimise work or organisational performance. All three methods were developed to identify safety risks; however, in the present study each method has demonstrated a capacity to identify risks to optimal performance, with a majority of the risks identified not relating to rider safety. Consequently, whilst further applications of the methods for RA purposes are recommended, exploration of using the methods to optimise work and organisational performance is warranted.

Third, the selection and use of STPA, EAST-BL, or Net-HARMS is largely dependent on the following factors: (i) the aims, scope, and scale of the broader project and analysis; (ii) the time and resources available to HFE and safety practitioners; (iii) previous experience and expertise, including 
the constituent materials, methods, and models that are required; and, (iv) the complexity and nature of the domain in which the analysis is being applied. This latter point is especially relevant to the current RA analysis as the goal was to identify risks that could degrade cycling team performance as opposed to those that would affect safe working practices (i.e., a more traditional application area for RA activities). Accordingly, we found that due to the terminology used in the STPA taxonomy, a greater number of safety-related risks that could indirectly impair optimal sports performance were identified, such as 'rider cannot control bike and crashes/falls/collides with other riders'. This is logical as STAMP and STPA were developed initially to support incident analysis and prevention in the context of systems engineering and development (Leveson 2004; Leveson 2011; Leveson et al. 2009). In terms of the broader HFE discipline, researchers and practitioners are encouraged to carefully consider the aims of the project and their competence and experience in using RA methods, particularly due to the time required in which to develop the models and networks of the target system which are required prior to the analyses.

Fourth, this study modified the original Net-HARMS method by stratifying tasks by agent type. Risk mode descriptions were merged if no differences between agents could be identified or were handled individually if there were differences for a given task. Future Net-HARMS applications are encouraged to examine risks separately according to the type of agents who are undertaking a given task where it is considered necessary to do so. Like much of the Net-HARMS analysis itself, the decision is subject to the expertise and domain knowledge of the analyst.

Fifth, three analysts (AH, SM, PS) independently performed the RA evaluations to avoid biasing the results. All three analysts have a background in sport and exercise science, as well as formal qualifications in sports performance and injury management and prevention. A high level of subject matter knowledge and domain expertise when undertaking the RA evaluations was - and arguably is for all RA evaluations in HFE - beneficial with regard to the identification of credible risks, as well as how those risks are to be articulated, interpreted, and described. Whilst a good RA method should, to an extent, drive the analysis and support the HFE practitioner to identify risks, it is helpful for analysts to have at least a basic albeit sufficient understanding of the system in question. It is for this reason 
that sports organisations would likely benefit from the expertise that a dedicated HFE professional could provide across all levels of the sports system, from the equipment, playing environment, and athlete level through to coaching, management, and club policy.

Sixth, whilst the analysis suggested that Net-HARMS is the most capable method under the proviso that the identified risks are credible, there is nevertheless a matter of having to go through thousands of potential risks and risk descriptions to otherwise locate the 'signal' amongst the 'noise'. In other words, there is the question of which risks are more likely to happen, and amongst those, which risks should be prioritised and taken most seriously? Accordingly, network analysis metrics and the assignment of probability ratings (see section 4.5 below) are useful ways of helping to draw attention to key tasks within the network as well as risks that could be most likely to occur.

Seventh, and with all of the above aspects in mind, including the frequency and types of risks identified through to reported usability of the RA approaches, the question left to ask is: which systems-based RA method is most appropriate for identifying risks in the area of elite sports performance? Under the assumption that the identified Net-HARMS risks are credible, and that sufficient time and resources are available to HFE researchers and practitioners, we feel that NetHARMS should be considered in future studies examining the risks that could degrade the performance of athletes and teams within complex sports systems. This is due to the fact that NetHARMS identified thousands of emergent risks across the cycling race system. Despite recommending the use of Net-HARMS, it is also reasonable to adopt a pragmatic approach to the selection and use of RA methods so long as a suitable justification for doing so is provided (i.e., consistent with project goals, projected timeframes, and resource constraints).

\subsection{Limitations and considerations}

There are four notable limitations to this study. First, a probability or criticality rating was not assigned to the identified risks or to the consequences (i.e., severity and impact) of those risks, respectively. For example, Net-HARMS supports a qualitive ordinal scale of low, medium, and high with regard to the assignment of probability and criticality ratings for both the task and emergent risk 
phases. In one of the first studies to use EAST-BL, a proposed intervention strategy and impact rating comprehensive comparison of methods like the one here and was ultimately designed to support the development of interventions. The assignment of a probability and criticality rating is based on expert judgement, such as feedback obtained via consultation with users from the target system, which can highlight risks that may be critical and therefore require greater attention in terms of proposed remedial measures. Likewise, network analysis can be applied to the task networks used in EAST-BL and Net-HARMS to compute a range of quantitative metrics, such as indegree or outdegree centrality to show tasks that have a relatively high influence on other tasks. Second, only the total number of risks were reported, and not those risks that may be considered 'unique'. Depending on the detail of each risk description, a varying degree of interpretation may be required to identify duplicate risks. Third, it was easier to compare and contrast the frequency and types of the risks identified using EAST-BL and Net-HARMS given that these two methods use the same task network. STPA takes a fundamentally different approach, and so whilst a comparison of risk frequencies produced satisfactory results, examining the types of risks identified was much more challenging. Forth and finally, whilst Net-HARMS is capable of identifying emergent risks to a factor of two, STPA and EAST-BL currently lack the capability to account for emergent risks. An area of future research for systems thinking-based RA methods could involve testing how these methods might be further developed to account for multiplicative risk effects.

\subsection{Conclusion}

Systems thinking-based RA methods attempt to identify the hazards and associated risks across all levels of a system that may lead to accidents and adverse incidents. The current methods comparison analysis has shown that systems thinking-based RA approaches are not only useful in terms of their capacity to identify a high number of potential risks, but also that they can be applied to identify the risks that could detrimentally impact optimal (sports) performance. This represents a deviation away from a traditional safety management context. The impetus for this work was attributable to the fact that systems HFE methods and approaches are increasingly being applied in the context of sport, and 
so there was a need to test, evaluate, and report on the use of systems thinking-based RA methods in this area as well. Based on the findings, it is concluded that whilst risk frequencies differed across the methods, the application of STPA, EAST-BL, and Net-HARMS in the context of cycling team performance appeared to generate meaningful insights that may help to optimise future race performances. It is also concluded that the selection and application of systems thinking-based RA methods should be determined and justified based on a number of key practical and logistical considerations, including the purpose of the project and analysis, the time and resources available to HFE and safety practitioners, prior experience and expertise, and also the domain that is subject to the RA evaluation. Whilst Net-HARMS identified thousands of emergent risks and is therefore recommended in future applications, a pragmatic and reasoned approach to RA methods selection and use is also encouraged. Further work is required to apply systems thinking-based RA methods and approaches in new contexts and report on the strengths and limitations in doing so.

\section{Funding}

This work was supported by an Australian Research Council (ARC) Discovery Project grant (grant number: DP180100806).

\section{Acknowledgements}

We would like to thank Dr Amanda Clacy for her input and help with data collection. 


\section{References}

Allison, C.K., Revell, K.M., Sears, R., Stanton, N.A. (2017). Systems Theoretic Accident Model and Process (STAMP) safety modelling applied to an aircraft rapid decompression event. Safety Science 98: $159-166$.

Annett, J., Duncan, K.D., Stammers, R., Grey, M.J. (1971). Task analysis. Department of Employment Training information paper 6, HMSO, London.

Atkinson, G., Reilly, T. (1995). Sport, leisure and ergonomics. Proceedings of the Third International Conference on Sport, Leisure and Ergonomics, School of Human Sciences, Liverpool John Moores University, Liverpool, UK, Taylor \& Francis.

Banks, V.A., Stanton, N.A. (2019). Analysis of driver roles: Modelling the changing role of the driver in automated driving systems using EAST. Theoretical Issues in Ergonomics Science 20(3): 284-300.

Clacy, A., Goode, N., Sharman, R., Lovell, G.P., Salmon, P. (2019). A systems approach to understanding the identification and treatment of sport-related concussion in community rugby union. Applied Ergonomics 80: 256-264.

Dallat, C., Salmon, P.M., Goode, N. (2017). Risky systems versus risky people: To what extent do risk assessment methods consider the systems approach to accident causation? A review of the literature. Safety Science 119: 266-279.

Dallat, C., Salmon, P.M., Goode, N. (2018). Identifying risks and emergent risks across sociotechnical systems: The NETworked hazard analysis and risk management system (NET-HARMS). Theoretical Issues in Ergonomics Science 19(4): 456-482. 
Embrey, D.E. (1986). SHERPA: A systematic human error reduction and prediction approach. Proceedings of the international topical meeting on advances in human factors in nuclear power systems.

Hulme, A., Stanton, N.A., Walker, G.H., Waterson, P., Salmon, P.M. (2019a). What do applications of systems thinking accident analysis methods tell us about accident causation? A systematic review of applications between 1990 and 2018. Safety science 117: 164-183.

Hulme, A., Thompson, J., Plant, K.L., Read, G.J.M., McLean, S., Clacy, A., Salmon, P.M. (2019b). Applying systems ergonomics methods in sport: A systematic review. Applied Ergonomics 80: 214225.

Hulme, A., McLean, S., Read, G.J.M., Dallat, C., Bedford, A., Salmon, P.M. (2019c). Sports organisations as complex systems: Using Cognitive Work Analysis to identify the factors influencing performance in an elite Netball organisation. Frontiers in Sports and Active Living 1: 56.

Hulme, A., Salmon, P.M., Nielsen, R.O., Read, G.J.M., Finch, C.F. (2017a). Closing Pandora's Box: Adapting a systems ergonomics methodology for better understanding the ecological complexity underpinning the development and prevention of running-related injury. Theoretical Issues in Ergonomics Science 18(4): 338-359.

Hulme, A., Salmon, P.M., Nielsen, R.O., Read, G.J.M., Finch, C.F. (2017b). From control to causation: Validating a 'complex systems model' of running-related injury development and prevention. Applied Ergonomics 65: 345-354.

Jenkins, D.P., Stanton, N.A., Walker, G.H. (2017). Cognitive work analysis: Coping with complexity, CRC Press. 
Kirwan, B., Ainsworth, L.K. (1992). A guide to task analysis: The task analysis working group, CRC press.

Klein, G.A., Calderwood, R., Macgregor, D. (1989). Critical decision method for eliciting knowledge. IEEE Transactions on systems, man, cybernetics 19(3): 462-472.

Lane, B.R., Salmon, P.M., Cherney, A., Lacey, D., Stanton, N.A. (2019). Using the Event Analysis of Systemic Teamwork (EAST) broken-links approach to understand vulnerabilities to disruption in a darknet market. Ergonomics 62(9): 1134-1149.

Leveson, N. (2004). A new accident model for engineering safer systems. Safety Science 42(4): $237-$ 270.

Leveson, N. (2011). Engineering a safer world: Systems thinking applied to safety, MIT press.

Leveson, N., Dulac, N., Marais, K., Carroll, J. (2009). Moving beyond normal accidents and high reliability organizations: A systems approach to safety in complex systems. Organization Studies 30(2-3): 227-249.

Leveson, N., Thomas, J.P. (2018). STPA handbook, Cambridge, MA, USA.

Macquet, A.-C., Ferrand, C., Stanton, N. (2015). Divide and rule: A qualitative analysis of the debriefing process in elite team sports. Applied Ergonomics 51: 30-38.

Macquet, A.-C., Fleurance, P. (2007). Naturalistic decision-making in expert badminton players. Ergonomics 50(9): 1433-1450. 
Mahajan, H.S., Bradley, T., Pasricha, S. (2017). Application of systems theoretic process analysis to a lane keeping assist system. Reliability Engineering \& System Safety 167: 177-183.

Mclean, S., Hulme, A., Mooney, M., Read, G.J.M., Bedford, A., Salmon, P.M. (2019a). A systems approach to performance analysis in women's netball: Using work domain analysis to model elite netball performance. Frontiers in psychology 10: 201.

McLean, S., Salmon, P.M., Gorman, A.D., Dodd, K., Solomon, C. (2019b). Integrating communication and passing networks in football using social network analysis. Science and Medicine in Football 3(1): 29-35.

McLean, S., Salmon, P.M., Gorman, A.D., Read, G.J.M., Solomon, C. (2017a). What's in a game? A systems approach to enhancing performance analysis in football. PloS one 12(2): e0172565$\mathrm{e} 0172565$.

McLean, S., Salmon, P.M., Gorman, A.D., Naughton, M., Solomon, C. (2017b). Do inter-continental playing styles exist? Using social network analysis to compare goals from the 2016 EURO and COPA football tournaments knock-out stages. Theoretical Issues in Ergonomics Science 18(4): 370-383.

Naikar, N. (2005). Work Domain Analysis: Theoretical concepts and methodology. Department of Defence, Australian Government: Air Operations Division, Defence Science and Technology Organisation.

Naikar, N., Moylan, A., Pearce, B. (2006). Analysing activity in complex systems with Cognitive Work Analysis: Concepts, guidelines and case study for control task analysis. Theoretical Issues in Ergonomics Science 7(4): 371-394. 
Rasmussen, J. (1997). Risk management in a dynamic society: A modelling problem. Safety Science 27(2-3): 183-213.

Rasmussen, J., Svedung, I. (2000). Proactive risk management in a dynamic society, Swedish Rescue Services Agency.

Reilly, T., Lees, A. (1984). Exercise and sports equipment: Some ergonomics aspects. Applied Ergonomics 15(4): 259-279.

Revell, K.M., Allison, C., Sears, R., Stanton, N.A. (2019). Modelling distributed crewing in commercial aircraft with STAMP for a rapid decompression hazard. Ergonomics 62(2): 156-170.

Rising, J.M., Leveson, N.G. (2018). Systems-Theoretic Process Analysis of space launch vehicles. Journal of Space Safety Engineering 5(3-4): 153-183.

Rokseth, B., Utne, I.B., Vinnem, J.E. (2018). Deriving verification objectives and scenarios for maritime systems using the systems-theoretic process analysis. Reliability Engineering \& System Safety 169: 18-31.

Salmon, P.M., Walker, G.H., M Read, G.J., Goode, N., Stanton, N.A. (2017a). Fitting methods to paradigms: Are ergonomics methods fit for systems thinking? Ergonomics 60(2): 194-205.

Salmon, P.M., Dallat, C., Clacy, A. (2017b). It's not all about the bike: distributed situation awareness and teamwork in elite women's cycling teams. Proceedings from Contemporary Ergonomics and Human Factors 240-248 Staverton Estate, Daventry, Northamptonshire, United Kingdom, April 27. 
Salmon, P.M., Lenne, M.G., Walker, G.H., Stanton, N.A., Filtness, A. (2014). Using the event analysis of systemic teamwork (EAST) to explore conflicts between different road user groups when making right hand turns at urban intersections. Ergonomics 57(11): 1628-1642.

Salmon, P.M., Macquet, A.-C. (2019). Human factors and ergonomics in sport and outdoor recreation: From individuals and their equipment to complex sociotechnical systems and their frailties. Applied Ergonomics 80: 209-213.

Salmon, P.M., Mclean, S.M., Dallat, C., Mansfield, N., Solomon, C., Hulme, A. (2020). Human factors and ergonomics in sport: Applications and future directions, CRC Press.

Salmon, P.M., Read, G.J.M., Stevens, N.J. (2016). Who is in control of road safety? A STAMP control structure analysis of the road transport system in Queensland, Australia. Accident Analysis \& Prevention 96: 140-151.

Salmon, P.M., Read, G.J.M., Walker, G.H., Goode, N., Grant, E., Dallat, C., Carden, T., Naweed, A., Stanton, N.A. (2018). STAMP goes EAST: Integrating systems ergonomics methods for the analysis of railway level crossing safety management. Safety Science 110: 31-46.

Salmon, P.M., Stanton, N.A., Walker, G.H., Jenkins, D. (2005). Analysing C4i activity: A review of the Event Analysis of Systemic Teamwork (EAST) methodology, Tech. rep. Human Factors Integration Defence Technology Centre, Somerset, UK.

Schmid, D., Stanton, N.A. (2018). How are laser attacks encountered in commercial aviation? A hazard analysis based on systems theory. Safety science 110: 178-191.

Schmid, D., Stanton, N.A. (2019). A future airliner's reduced-crew: Modelling pilot incapacitation and homicide-suicide with systems theory. Human-Intelligent Systems Integration 1(1): 27-42. 
Shepherd, A. (1998). HTA as a framework for task analysis. Ergonomics 41(11): 1537-1552.

Stanton, N., Salmon, P., Walker, G.H. (2018). Systems thinking in practice: The event analysis of systemic teamwork.

Stanton, N.A. (2006). Hierarchical task analysis: Developments, applications, and extensions. Applied Ergonomics 37(1): 55-79.

Stanton, N.A. (2014). Representing distributed cognition in complex systems: How a submarine returns to periscope depth. Ergonomics 57(3): 403-418.

Stanton, N.A. (2016). On the reliability and validity of, and training in, ergonomics methods: A challenge revisited. Theoretical Issues in Ergonomics Science 17(4): 345-353.

Stanton, N.A., Baber, C., Harris, D. (2008). Modelling command and control: Event analysis of systemic teamwork, CRC Press.

Stanton, N.A., Harvey, C. (2017). Beyond human error taxonomies in assessment of risk in sociotechnical systems: A new paradigm with the EAST 'broken-links' approach. Ergonomics 60(2): 221-233.

Stanton, N.A., Salmon, P.M., Rafferty, L.A., Walker, G.H., Baber, C., Jenkins, D.P. (2017a). Human factors methods: A practical guide for engineering and design, CRC Press.

Stanton, N.A., Salmon, P.M., Walker, G.H. (2017b). Editorial New paradigms in ergonomics. Ergonomics 60(1): 151-156. 
Stanton, N.A., Salmon, P.M., Walker, G.H., Stanton, M. (2019a). Models and methods for collision analysis: A comparison study based on the Uber collision with a pedestrian. Safety Science 120: 117128.

Stanton, N.A., Salmon, P.M., Walker, G.H. (2019b). Systems thinking in practice: Applications of the Event Analysis of Systemic Teamwork (EAST) method. Boca Raton, CRC Press.

Vicente, K.J. (1999). Cognitive work analysis: Toward safe, productive, and healthy computer-based work, CRC Press.

Walker, G.H., Salmon, P.M., Bedinger, M., Stanton, N.A. (2017). Quantum ergonomics: Shifting the paradigm of the systems agenda. Ergonomics 60(2): 157-166. 


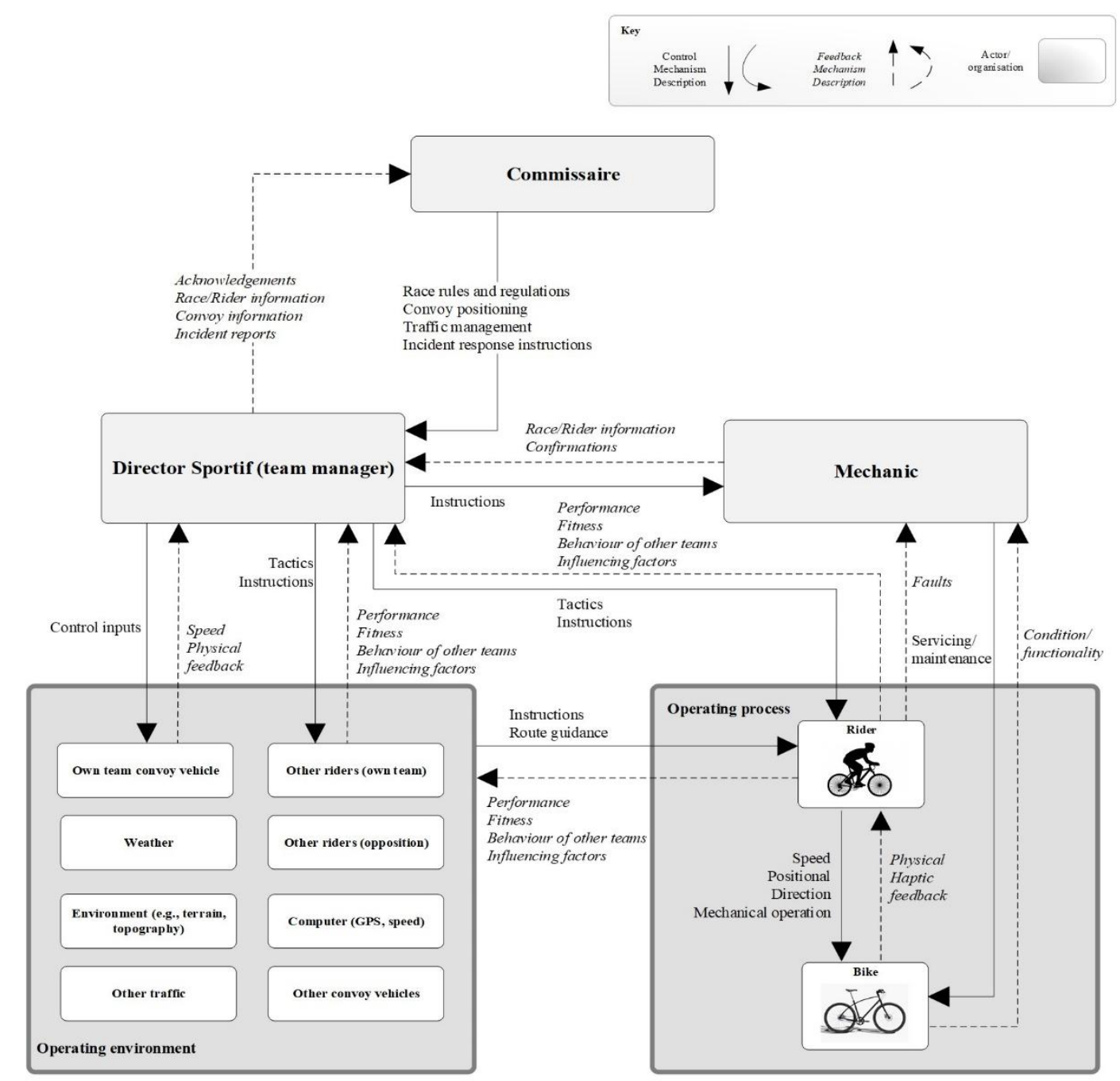

Figure 1: The STAMP control structure model of the cycling race system. 


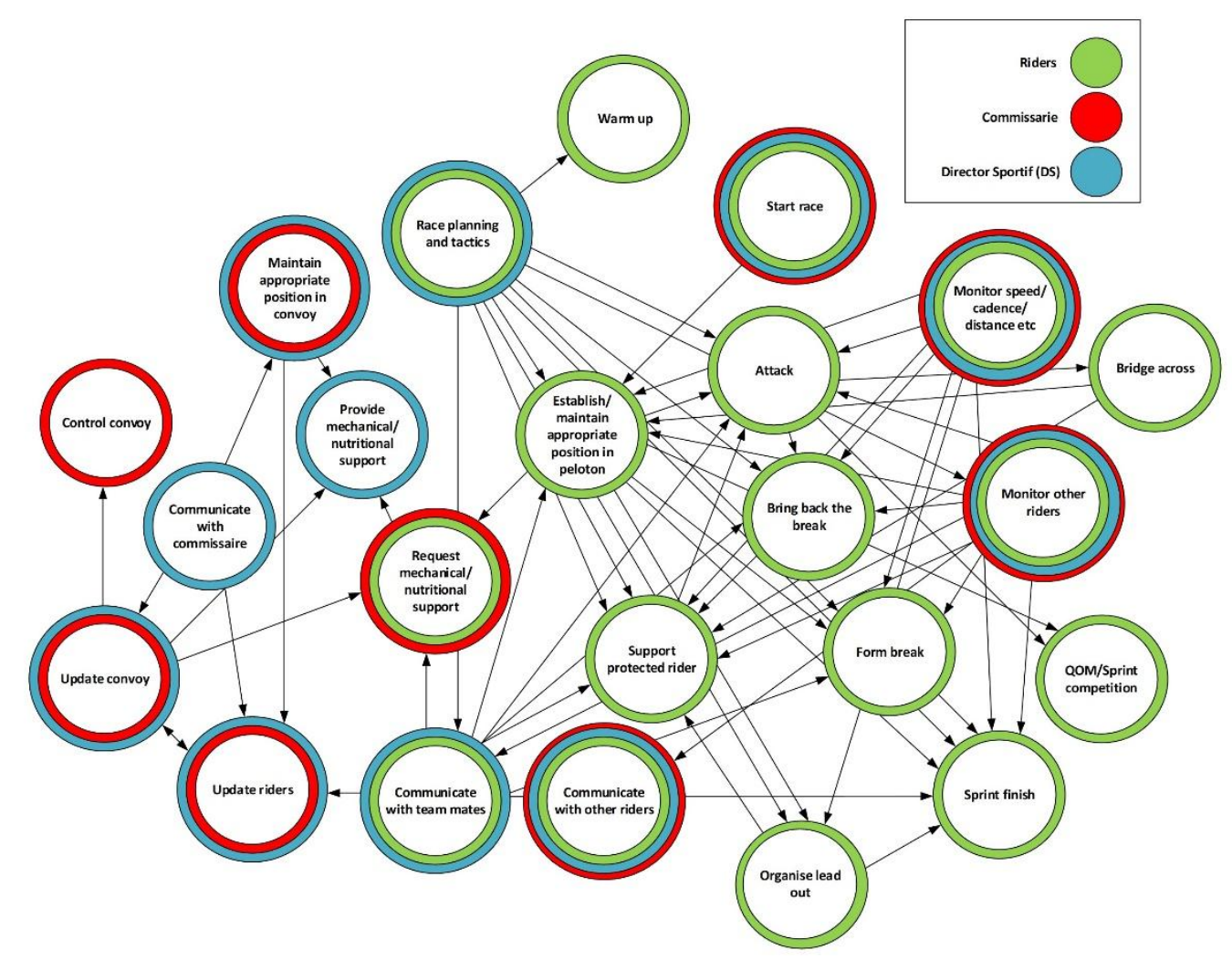

Figure 2: The task network of the cycling race system. Adapted from Salmon et al. (2017b). 


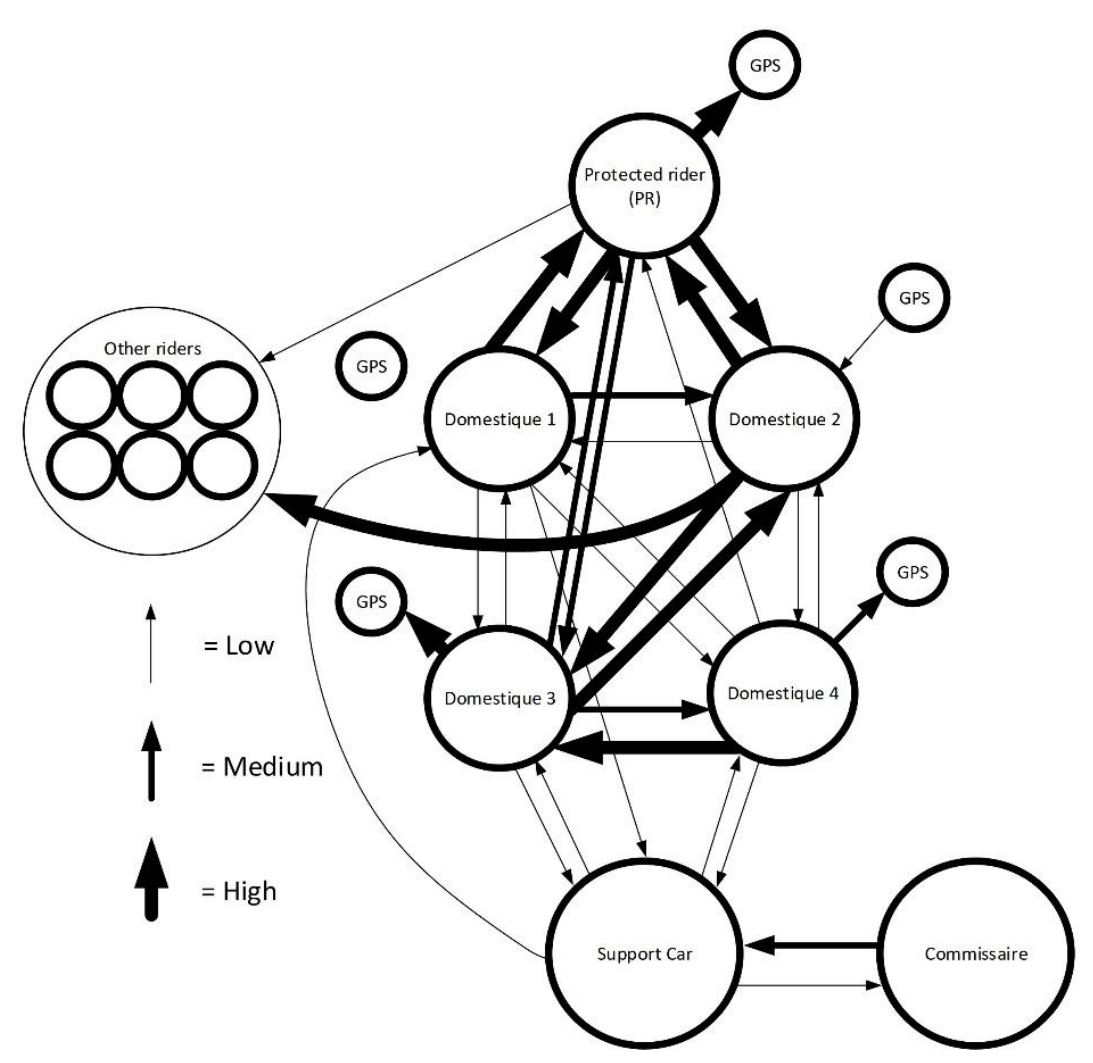

Figure 3: Social network for the cycling race system. Adapted from Salmon et al. (2017b). 


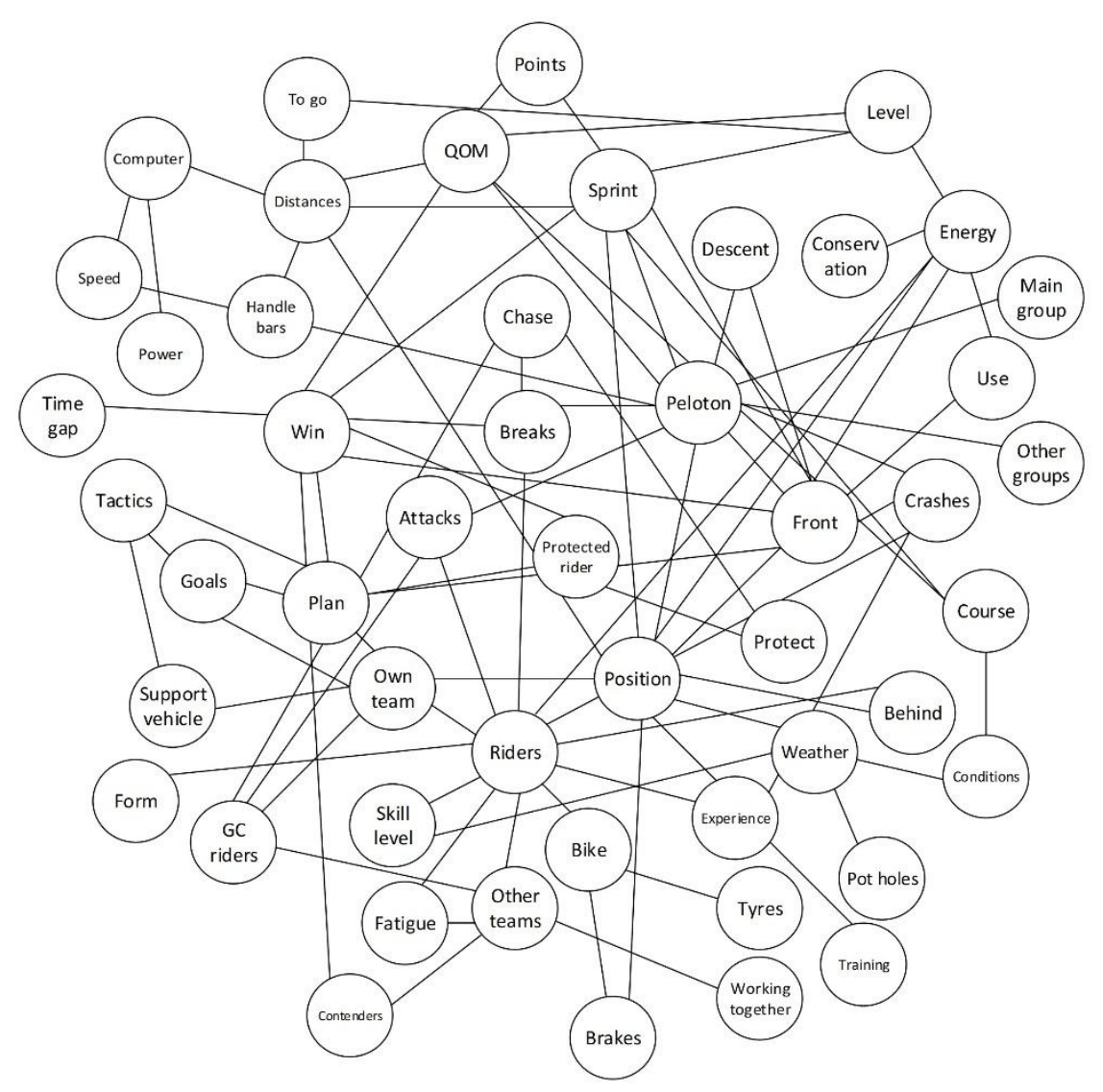

Figure 4: Information network for the cycling race system. Adapted from Salmon et al. (2017b). 


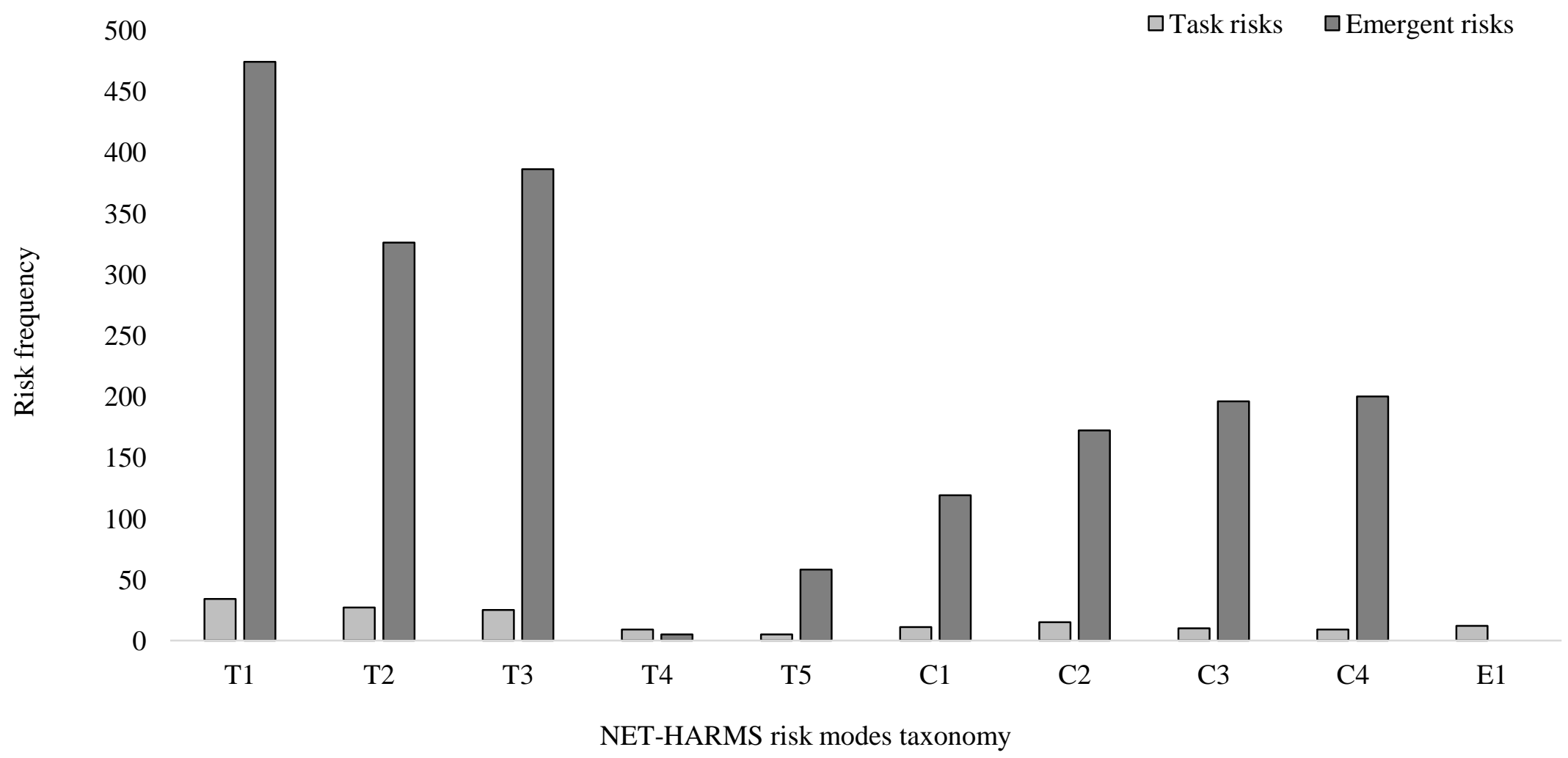

Figure 5: Frequency of the identified risks for both NET-HARMS phases (i.e., task risks and emergent risks) across three categories and 10 risk modes. 


\begin{tabular}{|c|c|c|c|c|c|c|c|c|c|c|c|c|c|c|c|c|c|c|c|c|c|c|c|c|c|c|c|}
\hline & Task & 1 & 2 & 3 & 4 & 5 & 6 & 7 & 8 & 9 & 10 & 11 & 12 & 13 & 14 & 15 & 16 & 17 & 18 & 19 & 20 & 21 & & 22 & 23 & & otal \\
\hline 1 & Warm up & & & & & & & & & & & & & & & & & & & & & & & & & & \\
\hline 2 & Race planning and tactics & $6 \quad 54$ & & & & & & $|114|$ & & $8 \quad 40$ & 01540 & & 938 & & & & & & & 1347 & 1039 & 93 & 357 & 46 & 1440 & 92 & 393 \\
\hline 3 & Maintain appropriate position in convoy & & & & & & 28 & & & & & & & & & & $\begin{array}{lll}1 & 8\end{array}$ & & & & & & & & & 3 & 316 \\
\hline 4 & Control convoy & & & & & & & & & & & & & & & & 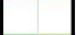 & & & & & & & & & & \\
\hline 5 & Update convoy & & & & $4 \quad 10$ & & 2213 & & & & & & & & & & $25 \mid$ & & 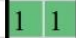 & & & & & & & 29 & 29 \\
\hline 6 & Update riders & & & & & 5 & 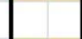 & & & & & & & & & & & & & & & 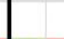 & & & & 5 & \\
\hline 7 & Communicate with team mates & & & & & & $6 \quad 13$ & & & & $\left|\begin{array}{ll}6 & 61\end{array}\right|$ & & & & & & & & $2 \quad 10$ & $0|760|$ & 470 & 56 & $60 \mid 5$ & & $6 \quad 60$ & 41 & 402 \\
\hline 8 & Communicate with other riders & & & & & & & & & & & & & & & & & & & & & 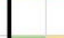 & & & & & \\
\hline 9 & Organise lead out & & & & & & & & & & $6 \quad 29$ & & & & & & & & & & & $4 \quad 1$ & 13 & & & 10 & 42 \\
\hline 10 & Sprint finish & & & & & & & & & & & & & & & & & & & & & & & & & & \\
\hline 11 & QOM/sprint competition & & & & & & & & & & & & & & & & & & & & & & & & & & \\
\hline 12 & Monitor other riders & & & & & & & 14 & 14 & & \begin{tabular}{|ll}
4 & 33
\end{tabular} & & & & & & & & & $\begin{array}{lll}7 & 33\end{array}$ & $4 \quad 19$ & $3 \quad 2$ & 215 & & $5 \quad 33$ & & 194 \\
\hline 13 & Bridge across & & & & & & & & & & & & & & & & & & & & $5 \quad 21$ & 42 & 22 & & & & 43 \\
\hline 14 & Monitor speed/cadence/distance etc & & & & & & & & & $4 \quad 64$ & $44 \mid \begin{array}{ll}7 & 65\end{array}$ & & & & & & & & & $2 \quad 67 \mid$ & $7 \begin{array}{lll}7 & 61\end{array}$ & 65 & $53 \mid 5$ & 66 & $\begin{array}{lll}7 & 67\end{array}$ & 38 & 443 \\
\hline 15 & Start race & & & & & & & & & & & & & & & & & & & & 25 & & & & & 2 & 5 \\
\hline 16 & Provide mechanical/nutritional support & & & & & & & & & & & & & & & & & & & & & & & & & & \\
\hline 17 & Communicate with commissaire & & & 110 & & $3 \quad 10$ & 0 & & & & & & & & & & & & & & & & & & & 5 & 30 \\
\hline 18 & Request mechanical/nutritional support & & & & & & & & & & & & & & & & $\left|\begin{array}{ll}5 & 11\end{array}\right|$ & & & & & & & & & 5 & 11 \\
\hline 19 & Attack & & & & & & & & & & & $5 \quad 22$ & 27 & $\left|\begin{array}{ll}8 & 22\end{array}\right|$ & & & & & & & & & 6 & 22 & & 26 & 66 \\
\hline 20 & Establish/maintain appropriate position in peloton & & & & & & & & & $7 \quad 32$ & $2 \begin{array}{lll}2 & 32\end{array}$ & $4 \quad 32$ & & & & & & & 114 & 4232 & & 42 & 25 & & $8 \quad 26$ & & 193 \\
\hline 21 & Support protected rider & & & & & & & 4 & & 4 & & & & & 2 & & & & & $3 \quad 27$ & & & & & & 13 & 27 \\
\hline 22 & Bring back the break & & & & & & & & & & & & & & & & & & & & & $5 \quad 2$ & 20 & & & 5 & 20 \\
\hline 23 & Form break & & & & & & & & & & 22 & & & & & & & & & & & & & & & & 22 \\
\hline
\end{tabular}

Figure 6: Adjacency matrix plot indicating the frequency with which risks were identified for the task-information phase of the EAST-BL analysis, and the emergent risk identification phase for Net-HARMS. Blank rows and columns indicate that the associated task is not influenced by, or does not influence, other tasks within the network. EAST-BL frequencies are positioned on the left of a given column whereas Net-HARMS frequencies are positioned to the right. Green (lighter) and red (darker) cell colours indicate lower and higher risk frequencies, respectively. The total EAST-BL and Net-HARMS risk frequencies adds up to 345 and 1,936 risks, respectively. 


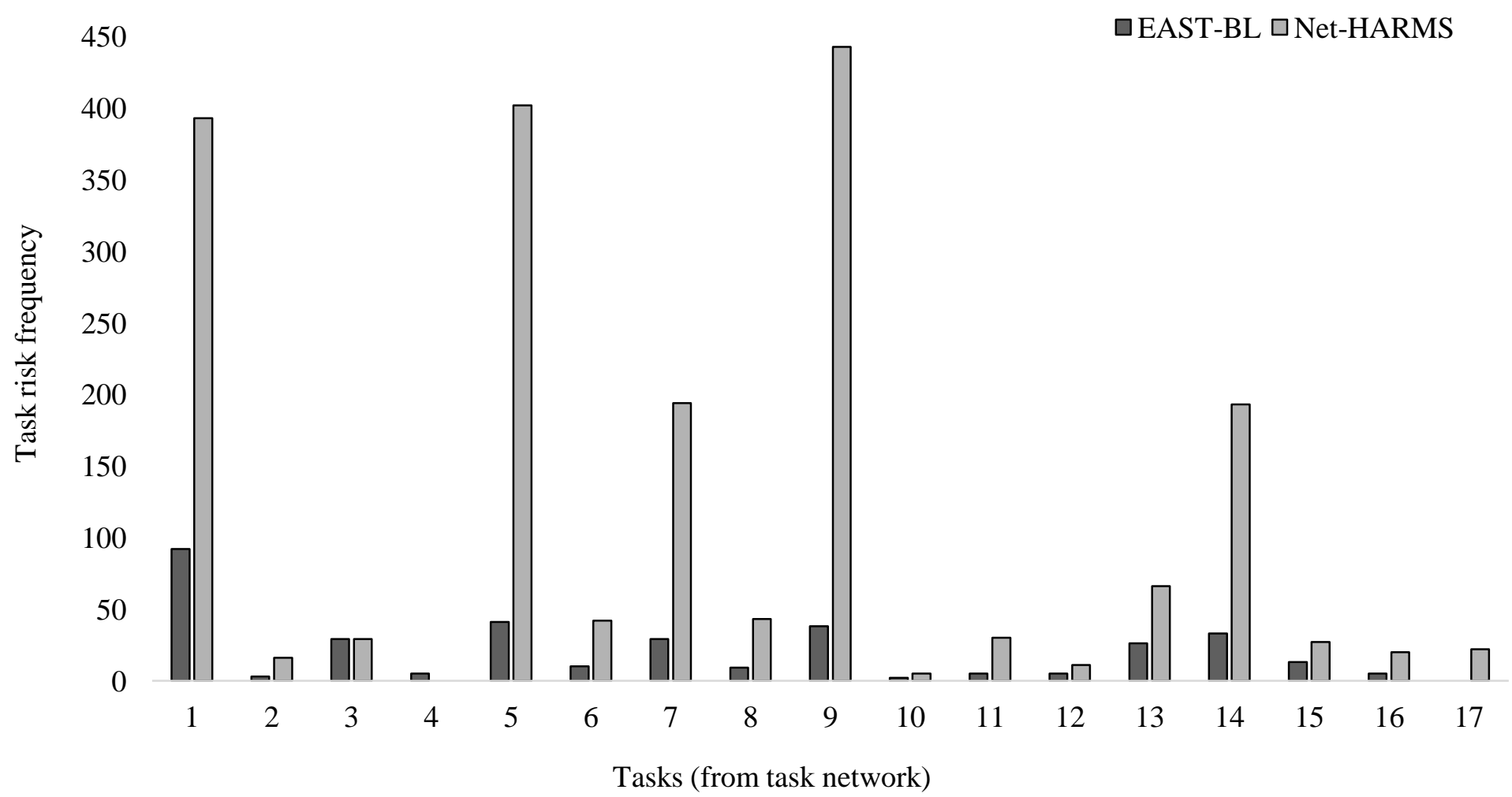

Figure 7: Differences between task risk frequencies (i.e., aggregated values across all linked tasks) for the EAST-BL and Net-HARMS analyses among tasks with outgoing relationships. 1, Race planning and tactics; 2, Maintain appropriate position in convoy; 3, Update convoy; 4, Update riders; 5, Communicate with team mates; 6, Organise lead out; 7, Monitor other riders; 8, Bridge across; 9, Start race; 10, Monitor speed/cadence/distance etc; 11, Communicate with commissaire; 12, Request mechanical/nutritional support; 13, Attack; 14, Establish/maintain appropriate position in peloton; 15, Support protected rider; 16, Bring back the break; 17, Form break 
Table 1: Excerpt of the STPA risk assessment analysis. For illustrative purposes only a select few examples are chosen for different agents and factors.

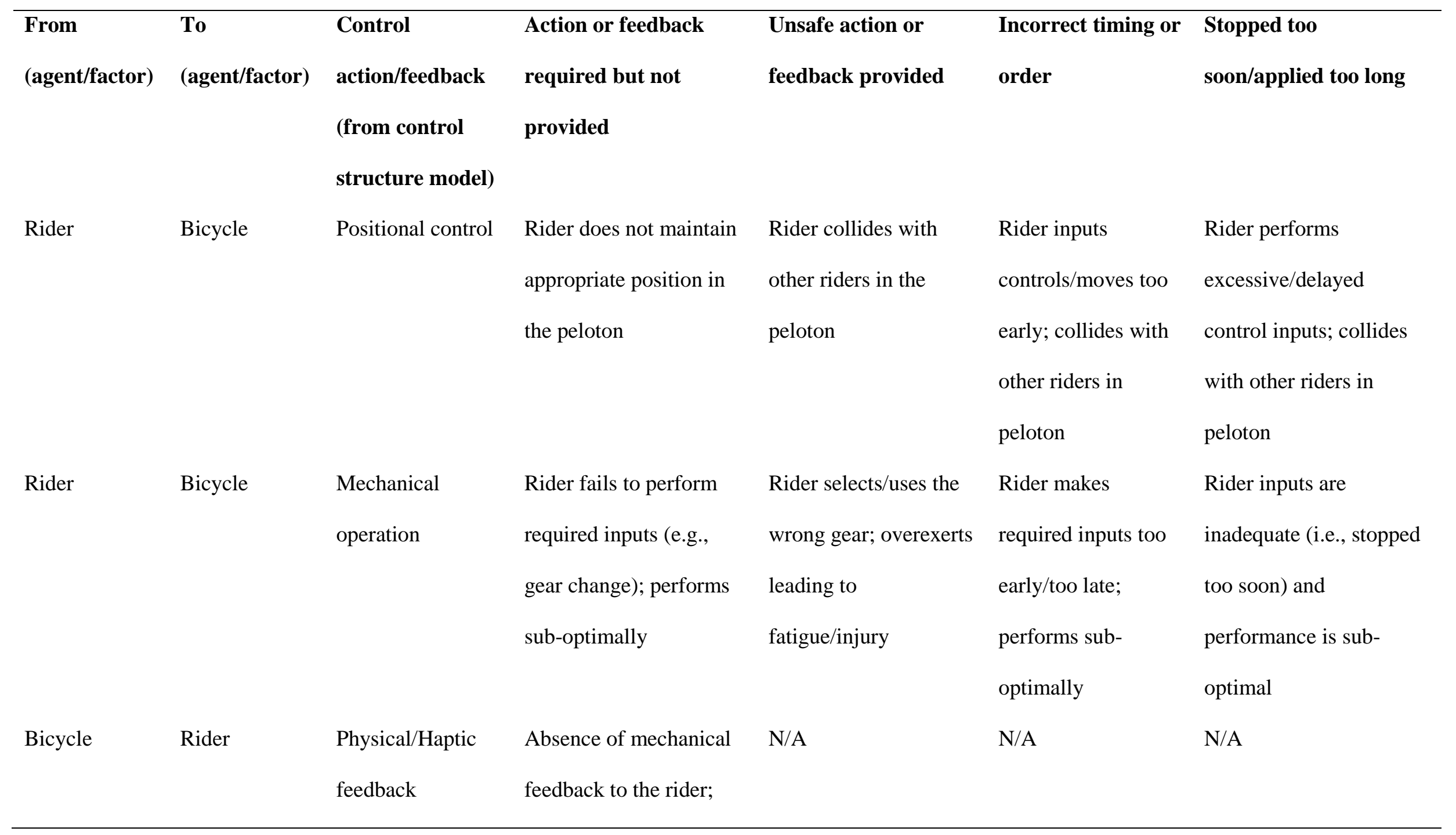




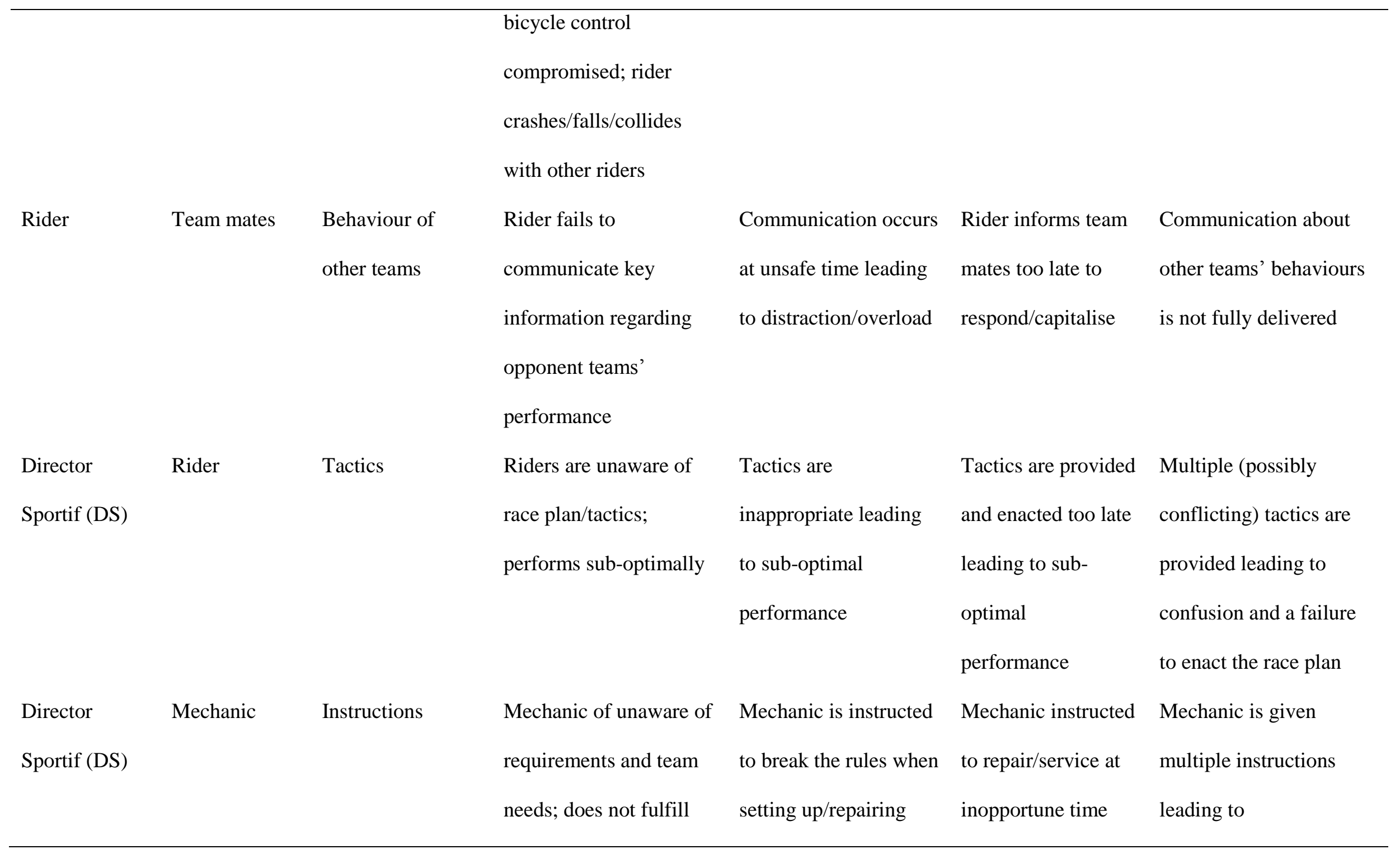




\begin{tabular}{|c|c|c|c|c|c|c|}
\hline & & & role leading to sub- & bikes leading to & & overload/confusion; \\
\hline & & & optimal performance & disqualification & & inappropriate response \\
\hline \multirow[t]{5}{*}{ Commissaire } & Director & Race rules and & DS does not fully & Commissaire distracts & Rules are enforced & Commissaire is too stric \\
\hline & Sportif (DS) & regulations & understand race rules & the DS from driving & too late; they are & in rule interpretation \\
\hline & & & and regulations leading & task leading to crash & violated/the team & creating/biased/unfair \\
\hline & & & to illegal behaviours & with rider/convoy & penalised & penalties \\
\hline & & & & vehicle & & \\
\hline
\end{tabular}

Table 2: Excerpt of the task-information EAST-BL risk assessment analysis for multiple linked tasks. For illustrative purposes only a single example of a risk is provided for a given task relationship.

\begin{tabular}{lll}
\hline From (task A) & To (task B) $\quad$ Outcome/risk description \\
\hline
\end{tabular}




$\begin{array}{lll}\text { Race planning and tactics } & \text { Warm up } & \text { Distances } \\ \text { Request } & \text { Provide } & \text { Bike } \\ \text { mechanical/nutritional } & \text { mechanical/nutritional } & \\ \text { support } & \text { support } & \\ \text { Establish/maintain } & \text { Sprint finish } & \text { Position } \\ \text { appropriate position in } & & \\ \text { peloton } & & \text { Tactics } \\ \text { Support protected rider } & \text { Organise lead out } & \\ \text { Monitor } & & \text { Computer } \\ \text { speed/cadence/distance etc. } & & \\ & & \end{array}$

\section{A)}

Distances

Distance covered in the warm up may be too long or too short, resulting

in sub optimal race performance (i.e., under prepared or physical over exertion)

The team DS/team mechanic are not aware of, or will not be able to provide support for, mechanical issues relating to bike and its components

Riders not aware of their current position within the peloton, leading to an inappropriate/suboptimal sprint finish

Tactics to facilitate an appropriate lead out are not communicated, the lead out for the protected rider is suboptimal/inadequate/poorly executed Riders not aware of essential feedback pertaining to race performance, including power and speed, and so closing the gap to the lead riders/breakaway is suboptimal/inadequate/performed inappropriately/misjudged 
Table 3: Excerpt of the NET-HARMS risk assessment analysis for the emergent risk phase. The initial task 'Race planning and tactics' and its associated risk T3 (i.e., task completed inadequately) from phase one is carried forwards and combined with the related task 'Warm up'. The NET-HARMS risk modes taxonomy is systematically applied again resulting, in the example provided, a further eight emergent risks. 


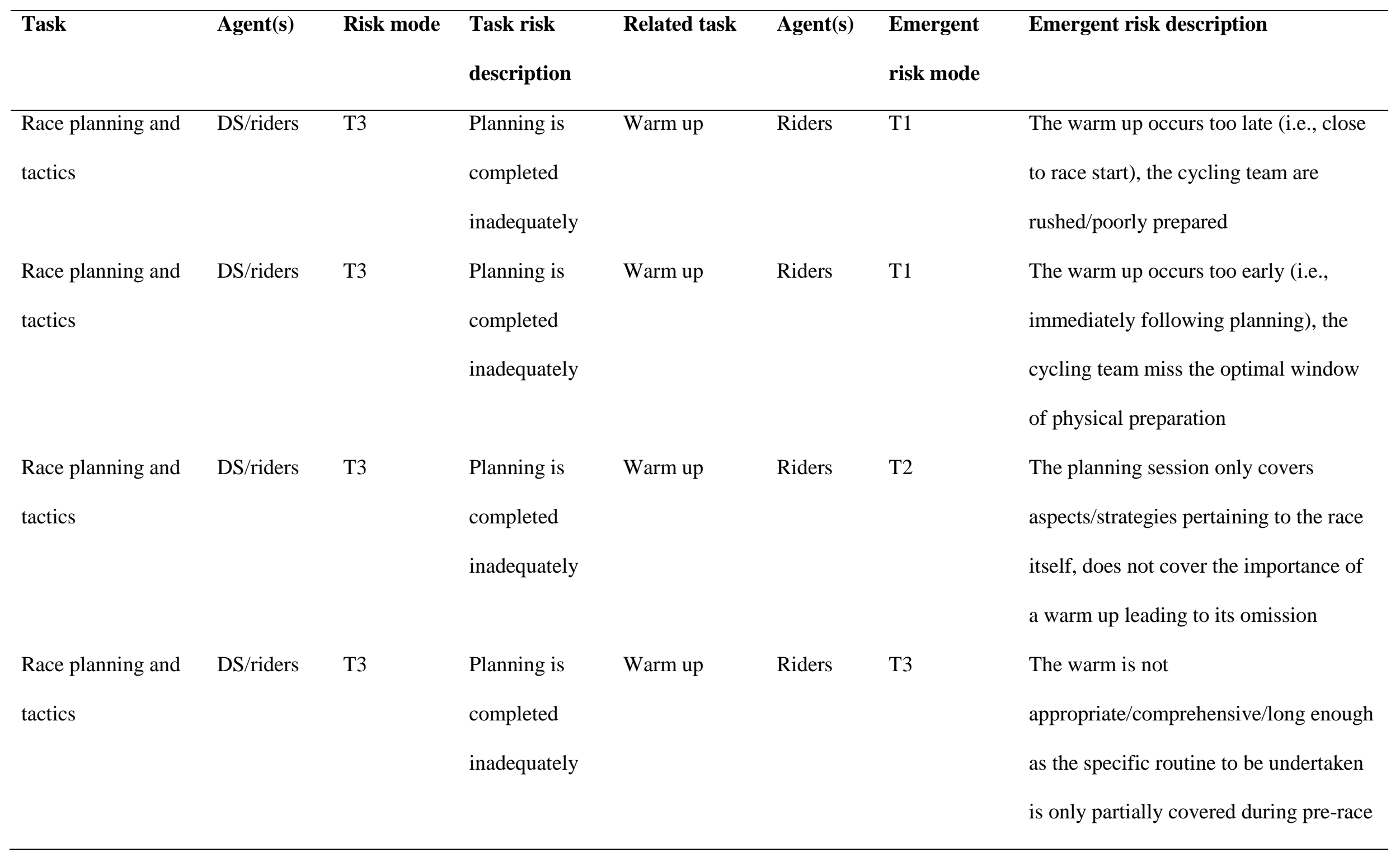




\begin{tabular}{|c|c|c|c|c|c|c|c|}
\hline & & & & & & & discussions \\
\hline Race planning and & DS/riders & $\mathrm{T} 3$ & Planning is & Warm up & Riders & $\mathrm{T} 5$ & The warm up is inappropriate (i.e., is \\
\hline \multirow[t]{3}{*}{ tactics } & & & completed & & & & excessive/physically \\
\hline & & & inadequately & & & & demanding/exertional/vigorous/too \\
\hline & & & & & & & extreme based on the conditions) \\
\hline \multirow[t]{3}{*}{ Race planning and } & DS/riders & $\mathrm{T} 3$ & Planning is & Warm up & Riders & $\mathrm{C} 1$ & Critical warm up-related information is \\
\hline & & & completed & & & & not communicated due to a \\
\hline & & & inadequately & & & & brief/insufficient planning session \\
\hline \multirow[t]{4}{*}{ Race planning and } & DS/riders & $\mathrm{T} 3$ & Planning is & Warm up & Riders & $\mathrm{C} 2$ & Due to inadequate planning/pre-race \\
\hline & & & completed & & & & discussions, the wrong information is \\
\hline & & & inadequately & & & & communicated/passed around the \\
\hline & & & & & & & cycling team \\
\hline \multirow[t]{4}{*}{ Race planning and } & DS/riders & $\mathrm{T} 3$ & Planning is & Warm up & Riders & $\mathrm{C} 3$ & Not all relevant information is \\
\hline & & & completed & & & & communicated to support the optimal \\
\hline & & & inadequately & & & & pre-race warm up routine as the \\
\hline & & & & & & & planning session is rushed/kept brief \\
\hline
\end{tabular}


Table 4: A comparison of the similarities and differences between STPA, EAST-BL, and Net-HARMS according to a set of devised theoretical and methodological constructs.

\section{Theory and principles}




\begin{tabular}{|c|c|c|c|}
\hline Control theory & $\checkmark$ & $x$ & $x$ \\
\hline Emergence & $x$ & $x$ & $\checkmark$ \\
\hline Taxonomy & $\checkmark$ & $x$ & $\checkmark$ \\
\hline Networks & $x$ & $\checkmark$ & $\checkmark$ \\
\hline Multiple system levels & $\checkmark$ & $\checkmark$ & $\checkmark$ \\
\hline Information elements & $\checkmark$ & $\checkmark$ & $\checkmark$ \\
\hline Hierarchical control structure & $\checkmark$ & $x$ & $x$ \\
\hline Tasks & $x$ & $\checkmark$ & $\checkmark$ \\
\hline Social agent & $\checkmark$ & $\checkmark$ & $\checkmark$ \\
\hline Feedback & $\checkmark$ & $\checkmark$ & $\checkmark$ \\
\hline
\end{tabular}


